\title{
Digital Data Storage Using DNA Nanostructures and Solid-State Nanopores
}

Kaikai Chen, ${ }^{+, \neq}$Jinglin Kong, ${ }^{\dagger, \neq}$ Jinbo Zhu, ${ }^{\dagger}$ Niklas Ermann, ${ }^{\dagger}$ Paul Predki, ${ }^{\S}$ and Ulrich F. Keysert,*

${ }^{\dagger}$ Cavendish Laboratory, University of Cambridge, JJ Thomson Avenue, Cambridge, CB3 OHE, United Kingdom

§Iridia, Inc. 3156 Lionshead Ave, Suite 1, Carlsbad, CA 92010, USA

*Email: ufk20@cam.ac.uk

†These authors contributed equally. 


\section{ABSTRACT}

Solid-state nanopores are powerful tools for reading out the three-dimensional shape of molecules allowing for the translation of molecular structure information into electric signals. Here, we show a high-resolution integrated nanopore system for identifying DNA nanostructures that has the capability of distinguishing attached short DNA hairpins with only a stem length difference of 8 bp along a DNA double strand named DNA carrier. Using our platform, we can read up to 112 DNA hairpins with a separating distance of 114 bp attached on a DNA carrier which carries digital information. Our encoding strategy allows for the creation of a library of molecules with a size of up to $5 \times 10^{\wedge} 33\left(2^{\wedge} 112\right)$ which is only built from a few hundred types of base molecules for data storage and has the potential to be extended by linking multiple DNA carriers. Our platform provides a nanopore- and DNA nanostructure-based data storage method with convenient access and the potential for miniature scale integration.

KEYWORDS: Solid-state nanopores, nanopore sensing, single-molecule, DNA storage, DNA nanotechnology

Nanopore-based single molecule sensing has demonstrated its applicability for the analysis of single molecules in electrolyte solutions. ${ }^{1,2}$ The principle relies on the modulation of ionic current flow as molecules pass through a nanopore driven by an applied electric field. ${ }^{3}$ The current signal reflects information about the passing objects, especially their three-dimensional shapes. ${ }^{4-6}$ Thus the nanopore sensing method directly transforms structural information into electric signals and can probe individual molecules at the single-molecule level giving access to analysis with high throughput. ${ }^{7}$ Sensors 
based on nanopores are especially attractive as they can be used for multiplexed sensing and integrated into low-volume fluidic chips, suitable for miniature portable devices reducing material requirements. ${ }^{8}$ In the literature, nanopores have shown the ability to detect objects distributed along a linear DNA strand, ${ }^{9-13}$ including DNA nanostructures and proteins, for DNA or protein sensing. ${ }^{14-16}$ The DNA nanostructures along a strand can be used to encode digital information. ${ }^{14}$ For their utility in data storage, the number of bits stored on one DNA strand can be improved by increasing the density of the structures. The foremost goal is the reduction of the size of the structures and their separating distance on the path to achieving realistic data storage applications.

Digital data can be directly stored in a DNA sequence at high data density. ${ }^{17,18}$ This method depends on the synthesis and sequencing of DNA molecules that need complex devices and high costs for large amounts of data. ${ }^{19-21}$ As an alternative, storage of information in three-dimensional DNA nanostructures is an attractive strategy ${ }^{22,23}$ which can be easily integrated with solid-state nanopores. Combined with DNA nanotechnology, nanopores can be used to build devices with exceptional readout capabilities.

In this study, we demonstrate a solid-state nanopore platform for digital data storage. The route is to use high-density DNA hairpins as digital bits along a strand named a DNA carrier. The carriers can be built by mixing DNA scaffolds and oligonucleotides to create a library of a large number of different DNA molecules to store information. We show our nanopore has the capability of detecting DNA hairpins down to a length of around $3 \mathrm{~nm}$ to decode the digital information. The mixing method for DNA structure synthesis and nanopore sensing allows high-density data storage and has great potential for miniature scale integration. 
First, we establish our system by building DNA hairpins as digital bits along DNA carriers and examine the sensing capability of our nanopore platform. We used an empirical approach to optimise the structure size while maximising the success rate of our nanopore measurements. The most important design goal was to ensure that the nanostructures generate observable secondary current drops while being small enough to ensure smooth DNA translocations. In previous studies structures consisting of $299 \mathrm{bp}$ protrusions $^{12}$ and a group of DNA dumbbells ${ }^{14}$ were measured with $10 \mathrm{~nm}$ and $14 \mathrm{~nm}$ diameter nanopores, respectively. These structures allow for easy detection but the data density is severely limited due to a bit size of up to $100 \mathrm{~nm}$. We started by testing $\sim 20 \mathrm{bp}$ ds DNA protrusions which turned out to be undetectable using our established $\sim 14 \mathrm{~nm}$ nanopores. ${ }^{24,25}$ As smaller nanopores increase the signal-to-noise ratio but may lead to stalled translocations ${ }^{26,27}$ we decided to decrease the diameter of our glass nanopores to $\sim 5 \mathrm{~nm}$. These smaller pores allowed for detection of dsDNA protrusions consisting of hairpins of less than $25 \mathrm{bp}$ and difference in stem length of $8 \mathrm{bp}$. In addition, translocations are observed with almost constant velocity which simplifies data analysis. In this paper we show that $5 \mathrm{~nm}$ glass nanopores are suitable for the detection of structures differing in molecular volume by $8 \mathrm{bp}$ with good success rate in a salt concentration of $4 \mathrm{M} \mathrm{LiCl}$. Future development of lower noise nanopores and machine learning ${ }^{28}$ will allow for further reduction of the bit size and increased data density.

We synthesised the DNA carrier with protruding DNA hairpins by hybridizing a 7228base single strand (linearised M13mp18 DNA,${ }^{14}$ New England Biolabs) as the scaffold and short oligonucleotides (Integrated DNA Technologies) with complementary sequences, as shown in Figure 1a. They were mixed at a ratio of $1 / 6-1 / 3$ (the scaffold to the 
oligonucleotides), annealed for $50 \mathrm{~min}$, and filtered to remove the excess oligonucleotides. ${ }^{14}$ The oligonucleotides consist of $38 \mathrm{bp}$ complementary strands and hairpin-linked strands. We built DNA hairpins with different stem lengths of 8 bp and 16 bp (Figure 1a). The full sequences are shown in Supporting Information Section 1. We fabricated nanopores by directly pulling quartz capillaries (outer diameter $0.5 \mathrm{~mm}$ and inner diameter $0.2 \mathrm{~mm}$ ) with a laser-heated pipette puller (P2000, Sutter Instrument). An example Scanning Electron Microscope (SEM) image of the glass nanopores with diameter down to $\sim 5 \mathrm{~nm}$ is shown in Figure $1 \mathrm{~b}$. The characterisation is shown in Supporting Information Section 2.

The glass nanopores were integrated into our PDMS chips (Figure 1b) and then filled with $4 \mathrm{M} \mathrm{LiCl}$ solution ${ }^{29}$. We diluted DNA samples into $4 \mathrm{M} \mathrm{LiCl}$ with final concentrations of $0.1-1 \mathrm{nM}$ and added them to the cis side (the chamber outside the capillary). $400 \mathrm{mV}$ voltage was applied to drive DNA molecules through nanopores (Figure 1c). The ionic current across the nanopore was recorded with an amplifier (Axopatch 200B, Molecular Devices), filtered with an external $49.9 \mathrm{kHz}$ Bessel filter (Frequency Devices) and digitised at a $250 \mathrm{kHz}$ sampling rate with a data card (PCI 6251, National Instruments). We used custom-made LabVIEW algorithms to collect the data and analyse the current traces to find single translocation signals (named events). Unfolded events ${ }^{30}$ were used to identify substructures with their positions along the linear strands. ${ }^{31}$ 


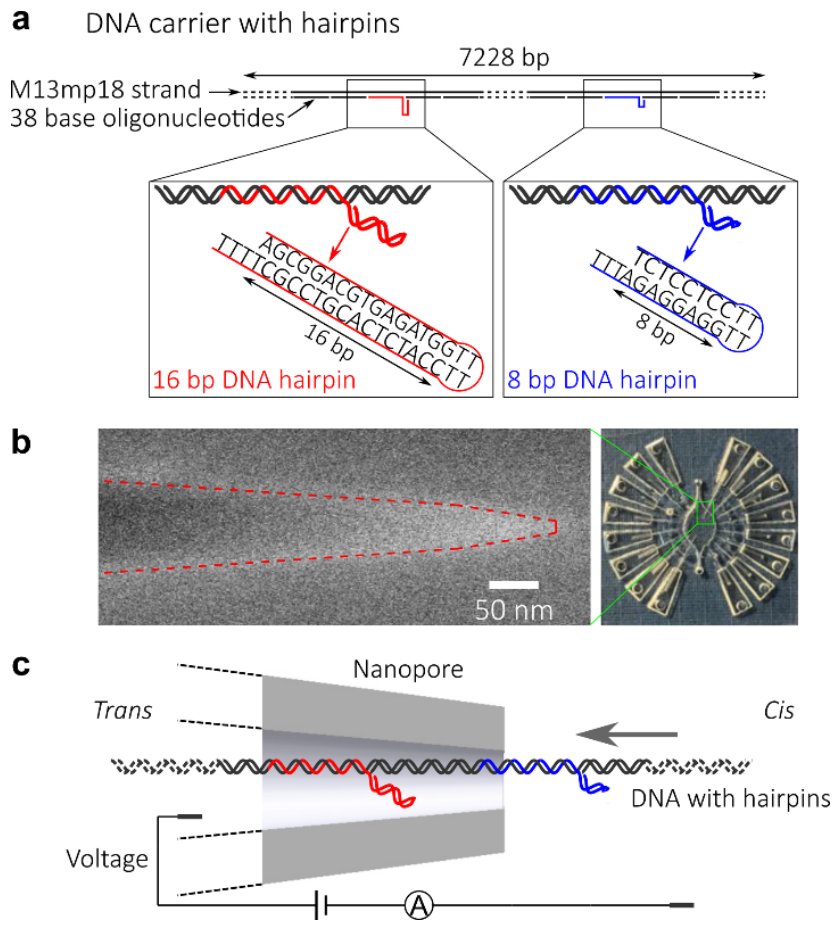

Figure 1. Design of DNA carriers with attached DNA hairpins and nanopore measurement. (a) A DNA carrier with $16 \mathrm{bp}$ (red) and $8 \mathrm{bp}$ (blue) DNA hairpins. Structures and sequences of the hairpins are shown at the bottom. (b) The SEM image of a glass nanopore and the integrated chip. Dashed lines indicate the outer edges (scale bar $=50 \mathrm{~nm}$ ). (c) Schematic of the measurement of the DNA carrier with a nanopore.

We tested the current and spatial resolution of our detection system with two DNA carrier designs and analysed the translocation events (see data analysis in Supporting Information Section 3). First, we measured DNA carriers with one 16 bp hairpin and one 8 bp hairpin on each (Figure 2a). Two example translocation events are shown with either end of the DNA entering the pore first. The current blockade $\Delta l_{0}$ caused by the doublestranded DNA backbone corresponds to the nearly constant level while the hairpin structures are indicated by secondary peaks. We analysed the secondary current drop $\Delta /$ for the 16 and $8 \mathrm{bp}$ hairpins and plotted the statistics in Figure 2a (right panel). As 
expected the Gaussian fits show that the 16 bp hairpin caused a deeper current drop than the $8 \mathrm{bp}$ hairpin. Our glass nanopore system is therefore able to distinguish the protruding hairpins with a size difference of only $8 \mathrm{bp}$ with data from tens of events. In the second design, we placed two $16 \mathrm{bp}$ long hairpins at a distance of $76 \mathrm{bp}$ to explore the minimum distance for bits that can be clearly distinguished with our nanopores (Figure $2 \mathrm{~b}$ ). We defined the resolved and unresolved events by analysing whether the peaks were separated in the time series with examples shown in Figure $2 \mathrm{~b}$. The fractions of resolved events for three independent pores show the two peaks were separated for nearly half of all unfolded events. The length of 76 base pairs is only $\sim 26 \mathrm{~nm}$, about ten times below the diffraction limit and comparable to the resolution limit of complex optical imaging systems. ${ }^{32}, 33$ Our nanopore platform shows the capability of identifying DNA nanostructures by their size even at small distances of structures.

a
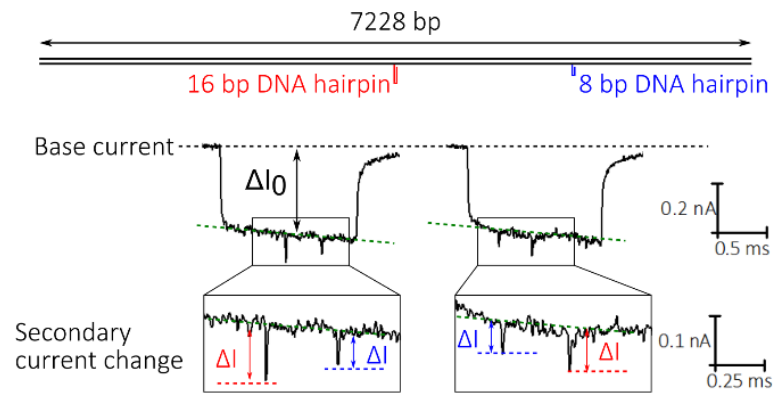

b
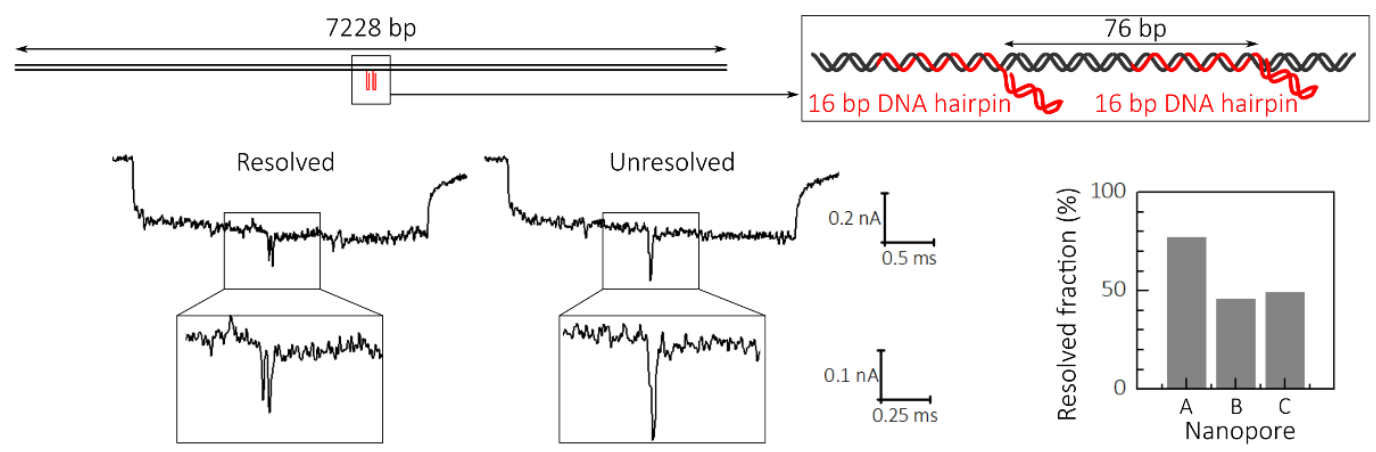

Figure 2. Resolution test of the nanopore system. (a) Discrimination of $16 \mathrm{bp}$ and $8 \mathrm{bp}$ DNA hairpins. The two hairpins are placed at $50 \%$ and $75 \%$ positions of the carrier. The 
secondary current changes in the sample events correspond to the two hairpins attached on the DNA carrier. The right panel shows the histogram of $\Delta /$ from 59 events. (b) Detection of two close hairpins. Two 16 bp hairpins are placed at the middle position with a distance of $76 \mathrm{bp}$. The revolved events show two separated peaks while the unresolved events show a single large peak. The fractions of resolved events measured with 3 distinct nanopores are shown in the right panel. The detailed designs with DNA sequences are shown in Supporting Information Section 1.

We then designed dense hairpin arrays on the carriers to encode digital information. In our design, an 8 bp hairpin encodes a digital bit ' 0 ' while a 16 bp hairpin a ' 1 '. The distance between bits is set to 114 bp to aid data analysis, and each DNA carrier accommodates 56 hairpins (Figure 3a). The detailed design and sequences are shown in Supporting Information Section 1. The sample was measured with the nanopores shown in Figure 1b. 56 peaks (bits) were fully resolved as shown in the example event in Figure 3b. Using the knowledge about the structure we decoded the information by analysing the current drop amplitudes using a Bayesian inference method $^{34}$ (see the description in Supporting Information Section 4). For the single event shown in Figure 3b, we detected 7 errors (marked in grey) in the 56 bits as expected due to the overlap in the current drops of the 16 and 8 bp hairpins (see Figure 2a).

More independent events of the same molecules were used to improve the accuracy of identification of each bit (see examples in Supporting Information Section 4). We aggregated statistics from 58 events and calculated the natural logarithm of the probability ratio of the respective bit being a ' 1 ' versus a ' 0 ': $\ln \left(\frac{P\left(H_{1, m} \mid\{D\}\right)}{P\left(H_{0, m} \mid\{D\}\right)}\right)$, where $m$ is the bit number 
(1-56). Figure 3c shows the analysed results where a large $\left|\ln \left(\frac{P\left(H_{1, m} \mid\{D\}\right)}{P\left(H_{0, m} \mid\{D\}\right)}\right)\right|$ indicates a high confidence in our estimate. The aggregated analysis of 58 events reduced the number of errors by more than half to only 2 . It should be noted that when aggregating the estimate for a particular bit using many events, we might use the values of neighbouring bits in some events where the orders of bits were not correctly counted, introducing a disturbance. This is also reflected by the high confidence with consecutive ' 0 ' or ' 1 ' and a lower confidence for alternating patterns of ' 0 ' and ' 1 '. The confidence in the bit estimation increased with more events used, as shown in Figure 3d, meaning that errors can be reduced by aggregating more events. However, the correct reading of such data can be improved through established encoding strategies such as sub blocks or checksums in the future. Data analysis relying on machine learning can also improve the decoding accuracy. 
a

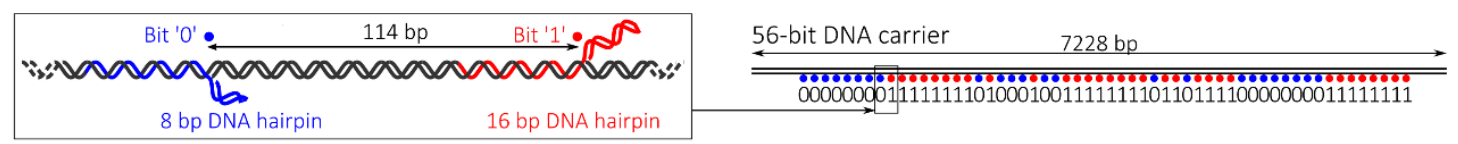

b

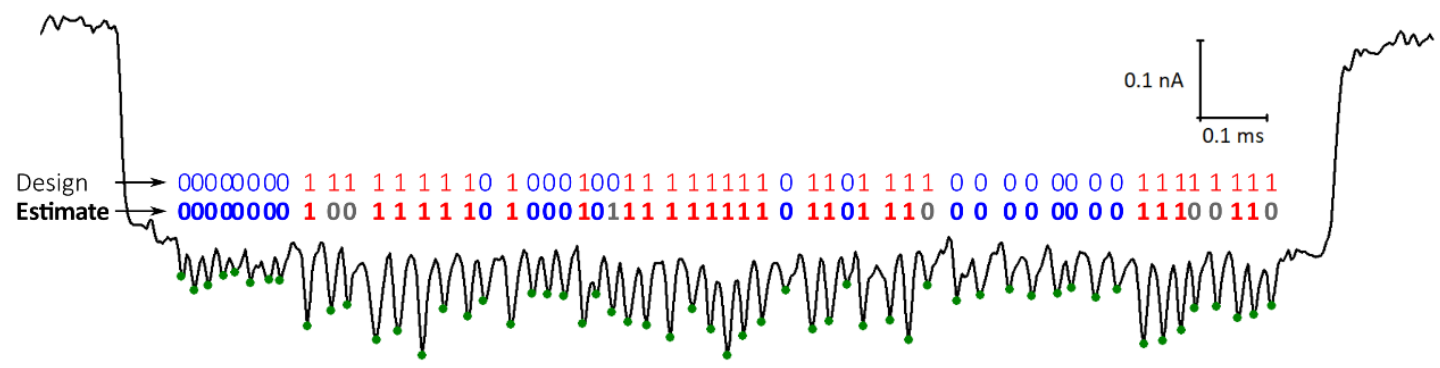

c

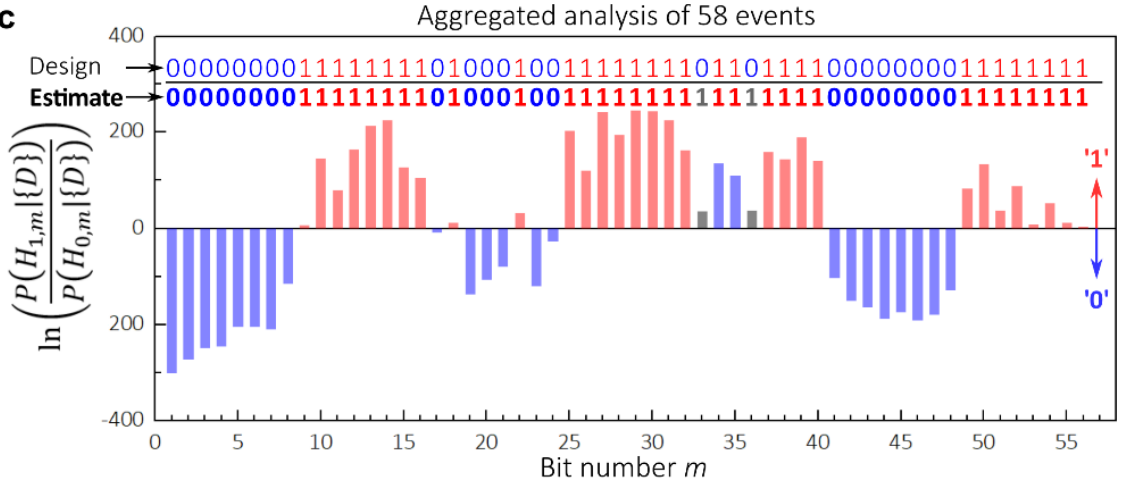

d

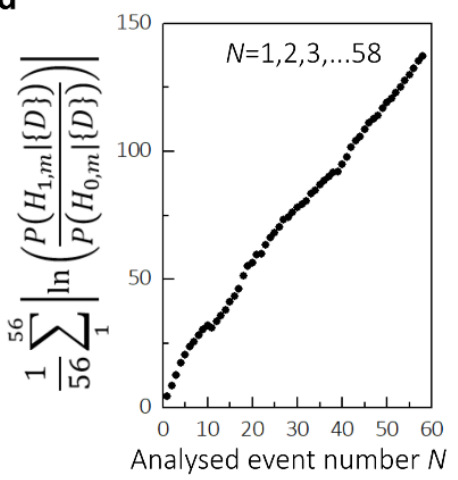

Figure 3. Design and decoding of a 56-bit DNA carrier. (a) Design of the 56-bit DNA carrier with 8 bp (blue) and 16 bp (red) DNA hairpins representing '0' and ' 1 ' respectively. (b) An example current signal measured with our nanopores. The grey values indicate misclassifications. (c) Information decoding by aggregating multiple events. The ' 0 ' and ' 1 ' were extracted by analysing 58 events. $\ln \left(\frac{P\left(H_{1, m} \mid\{D\}\right)}{P\left(H_{0, m} \mid\{D\}\right)}\right)$ shows the log ratio of the probability of a bit (at number $m$ ) being a ' 1 ' versus a ' 0 '. (d) Confidence of estimation as a function of analysed event number. $\frac{1}{56} \sum_{1}^{56}\left|\ln \left(\frac{P\left(H_{1, m} \mid\{D\}\right)}{P\left(H_{0, m} \mid\{D\}\right)}\right)\right|$ shows the average confidence values of the 56 bits.

With even smaller nanopores ${ }^{35-37}$ and nanopores on 2D materials ${ }^{38,39}$, one can decrease bit size and consequently increase the data density. In the current stage of the development, our 56-bit DNA carrier allows for libraries with $\sim 7.2 \times 10^{\wedge} 16\left(2^{\wedge} 56\right)$ different 
molecules with the possibility to encode data by mixing the scaffold and hundreds of oligonucleotides. The data density could be further enhanced by implementing a system with more bits with three or more corresponding current levels. Another route to increase storage capacity is to use longer DNA molecules with more bits.

To this end, we now show the possibility of expanding the bit number on a DNA carrier by linking 7228 bp DNA carriers using complementary single-stranded ends. The design of a dimer and one example event are shown in Figure 4a and Figure 4b. For this designed carrier, we had 12 errors out of the 112 bits by aggregating data from 19 events (marked in grey, Figure 4c). It should be noted that using this strategy, one can significantly increase the number of bits encoded on a DNA strand for larger libraries with $2^{\wedge}(56 \times n)$ different molecules, where $n$ is the number of monomers linked together. This linking method shows the potential of expanding the maximum information stored using our system. 
a

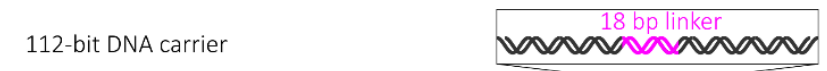

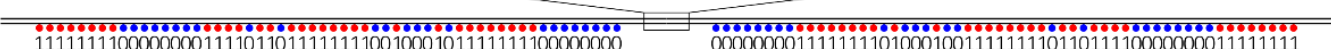
0000000111111110100010011111111011011110000000011111111

b

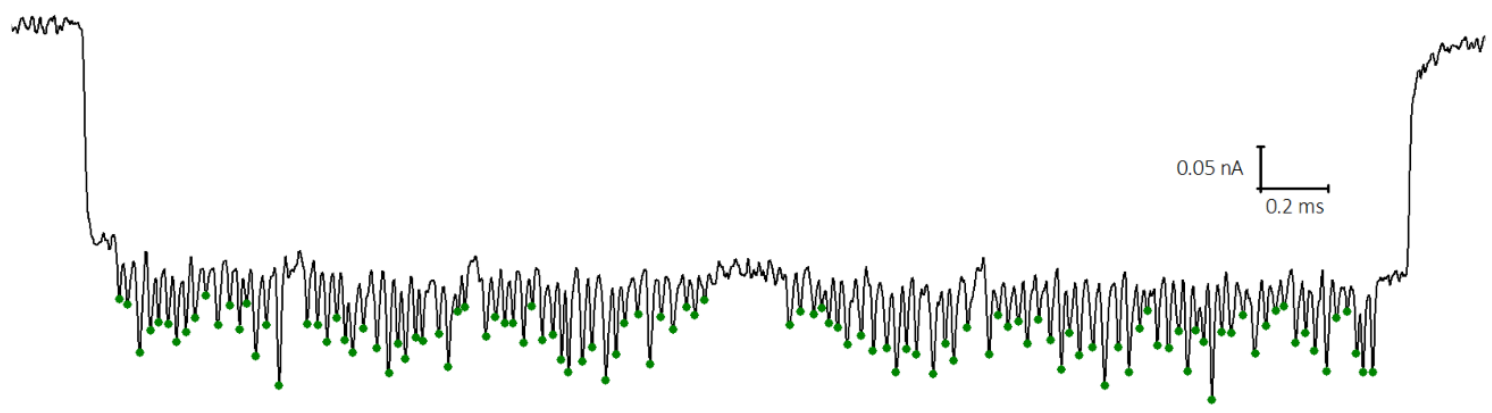

C

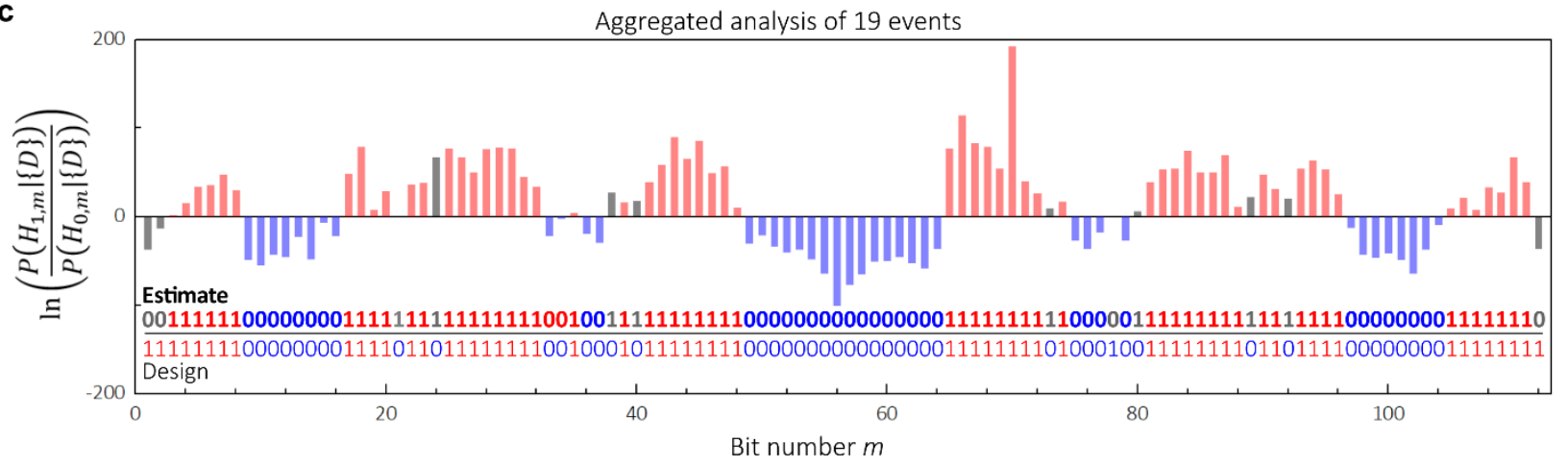

Figure 4. Extendibility of the storage system by linking 7228 bp DNA carriers. (a) Design of a dimer carrying 112-bit information by linking two 7228 bp carriers. (b) An example event caused by the dimer in the nanopore measurement. Green dots show recognised peaks in the analysis. (c) Aggregated analysis of 19 events for decoding the 112-bit information. Grey estimate values show the errors in our estimation (bold font) compared to the design.

To conclude, we developed a platform based on the combination of nanopore sensing and DNA nanotechnology for digital data storage. Our solid-state nanopore platform outperforms previous ones in identifying DNA nanostructures along a DNA strand in terms of resolution and data quality. We were able to detect short $16 \mathrm{bp}$ and 8 bp DNA hairpins and even distinguish between them on the same molecule. Hairpins with a distance of 
114 bp were clearly separated in current signals of nanopore measurements and could be resolved with a distance of $76 \mathrm{bp}$ in up to $\sim 50 \%$ of the events. We built 56 hairpins on a 7228 bp DNA carrier to store 56-bit information and used a Bayesian inference method with tens of events to reduce the reading error to 2 out of 56 bits. By linking two $7228 \mathrm{bp}$ carriers we encoded 112-bit data onto a single DNA molecule to show the potential of reading longer molecules.

Our digital data storage method shows an alternative to information storage in the DNA base sequence. It has advantages due to the ease of encoding and decoding by combining the simple mixing of oligonucleotides with nanopore sensing, which avoids complex devices and the use of enzymes and can be integrated at miniature scale. In future work, the technique can be improved in a number of ways. For instance, scalable synthesis of carriers can be achieved with micro- and nanofluidics to build a large number of DNA carriers to encode data with the mixing method. ${ }^{40}$ The nanopore decoding errors can be reduced by using encoding strategies and further optimising the size of DNA nanostructures and nanopores when parallel measurement systems become available. Even smaller nanopores should allow the design of DNA carriers with more densely spaced bits and more than two different hairpin lengths can be used to encode three or more values per position. Our platform is readily extensible and shows great potential for the integration of molecular information storage into nanoscale systems.

\author{
ASSOCIATED CONTENT
}

\title{
Supporting Information
}


Details on the synthesis and design of DNA carriers, nanopore characterization and measurement, data analysis for the DNA carriers with two hairpins, and data analysis for the 56-bit and 112-bit DNA carriers (PDF).

\author{
AUTHOR INFORMATION
}

Corresponding Author

*Email:ufk20@cam.ac.uk

\title{
Author Contributions
}

The manuscript was written through contributions of all authors. All authors have given approval to the final version of the manuscript. ¥These authors contributed equally.

\section{Notes}

The authors declare the following competing financial interest: the study was partly supported by Iridia Inc. through funding of the postdoc position of Dr Jinglin Kong.

\section{ACKNOWLEDGMENTS}

We thank the lab of Mark Howarth at Oxford University for kindly providing monovalent streptavidin. The authors thank Dr K. Misiunas, Dr J. Cama, and Dr A. Thorneywork for discussions and suggestions. K.C. and U.F.K. acknowledge funding from an ERC Consolidator Grant (Designerpores No. 647144). J.K. acknowledges funding from Cambridge Trust and Iridia Inc.. J.Z. acknowledges funding from an EPSRC grant (EP/M008258/1). N.E. acknowledges funding from the EPSRC, Cambridge Trust and Trinity Hall, Cambridge. 


\section{REFERENCES}

1. Storm, A. J.; Chen, J. H.; Ling, X. S.; Zandbergen, H. W.; Dekker, C. Nat. Mater. 2003, 2, (8), 537-40.

2. Li, J.; Gershow, M.; Stein, D.; Brandin, E.; Golovchenko, J. A. Nat. Mater. 2003, 2, (9), 611-5.

3. Dekker, C. Nat. Nanotechnol. 2007, 2, (4), 209.

4. Waduge, P.; Hu, R.; Bandarkar, P.; Yamazaki, H.; Cressiot, B.; Zhao, Q.; Whitford, P. C.; Wanunu, M. ACS Nano 2017, 11, (6), 5706-5716.

5. Yusko, E. C.; Bruhn, B. R.; Eggenberger, O. M.; Houghtaling, J.; Rollings, R. C.; Walsh, N. C.; Nandivada, S.; Pindrus, M.; Hall, A. R.; Sept, D.; Li, J.; Kalonia, D. S.; Mayer, M. Nat. Nanotechnol. 2017, 12, (4), 360-367.

6. $\quad H u$, R.; Rodrigues, J. V.; Waduge, P.; Yamazaki, H.; Cressiot, B.; Chishti, Y.; Makowski, L.; Yu, D.; Shakhnovich, E.; Zhao, Q.; Wanunu, M. ACS Nano 2018, 12, (5), 4494-4502.

7. Gooding, J. J.; Gaus, K. Angew. Chem. Int. Ed. 2016, 55, (38), 11354-11366.

8. Mikheyev, A. S.; Tin, M. M. Mol. Ecol. Resour. 2014, 14, (6), 1097-1102.

9. $\quad$ Singer, A.; Wanunu, M.; Morrison, W.; Kuhn, H.; Frank-Kamenetskii, M.; Meller, A. Nano Lett. 2010, 10, (2), 738-742.

10. Bell, N. A.; Keyser, U. F. J. Am. Chem. Soc. 2015, 137, (5), 2035-41.

11. Plesa, C.; Ruitenberg, J. W.; Witteveen, M. J.; Dekker, C. Nano Lett. 2015, 15, (5), 3153-8.

12. Plesa, C.; van Loo, N.; Ketterer, P.; Dietz, H.; Dekker, C. Nano Lett. 2015, 15, (1), 732-7.

13. Bulushev, R. D.; Marion, S.; Petrova, E.; Davis, S. J.; Maerkl, S. J.; Radenovic, A. Nano Lett. 2016, 16, (12), 7882-7890.

14. Bell, N. A. W.; Keyser, U. F. Nat. Nanotechnol. 2016, 11, (7), 645-651.

15. Sze, J. Y. Y.; Ivanov, A. P.; Cass, A. E. G.; Edel, J. B. Nat. Commun. 2017, 8, (1), 1552.

16. Zahid, O. K.; Wang, F.; Ruzicka, J. A.; Taylor, E. W.; Hall, A. R. Nano Lett. 2016, 16, (3), 2033-9.

17. Cox, J. P. Trends Biotechnol. 2001, 19, (7), 247-250.

18. Bancroft, C.; Bowler, T.; Bloom, B.; Clelland, C. T. Science 2001, 293, (5536), 1763-1765.

19. Goldman, N.; Bertone, P.; Chen, S.; Dessimoz, C.; LeProust, E. M.; Sipos, B.; Birney, E. Nature 2013, 494, (7435), 77-80.

20. Erlich, Y.; Zielinski, D. Science 2017, 355, (6328), 950-954.

21. Organick, L.; Ang, S. D.; Chen, Y.-J.; Lopez, R.; Yekhanin, S.; Makarychev, K.; Racz, M. Z.; Kamath, G.; Gopalan, P.; Nguyen, B. Nat. Biotechnol. 2018, 36, (3), 242.

22. Tumpane, J.; Sandin, P.; Kumar, R.; Powers, V. E. C.; Lundberg, E. P.; Gale, N.; Baglioni, P.; Lehn, J.-M.; Albinsson, B.; Lincoln, P.; Wilhelmsson, L. M.; Brown, T.; Nordén, B. Chem. Phys. Lett. 2007, 440, (1-3), 125-129.

23. Chandrasekaran, A. R.; Levchenko, O.; Patel, D. S.; Maclsaac, M.; Halvorsen, K. Nucleic Acids Res. 2017, 45, (19), 11459-11465.

24. Kong, J.; Zhu, J.; Keyser, U. F. Chem. Commun. 2017, 53, (2), 436-439. 
25. Kong, J.; Zhu, J.; Chen, K.; Keyser, U. F. Adv. Funct. Mater. 2018, 1807555.

26. Beamish, E.; Tabard-Cossa, V.; Godin, M. ACS Sensors 2017, 2, (12), 18141820.

27. Karau, P.; Tabard-Cossa, V. ACS Sensors 2018, 3, (7), 1308-1315.

28. Misiunas, K.; Ermann, N.; Keyser, U. F. Nano Lett. 2018.

29. Kowalczyk, S. W.; Wells, D. B.; Aksimentiev, A.; Dekker, C. Nano Lett. 2012, 12, (2), 1038-1044.

30. Mihovilovic, M.; Hagerty, N.; Stein, D. Phys. Rev. Lett. 2013, 110, (2), 028102.

31. Chen, K.; Juhasz, M.; Gularek, F.; Weinhold, E.; Tian, Y.; Keyser, U. F.; Bell, N.

A. W. Nano Lett. 2017, 17, (9), 5199-5205.

32. Jeffet, J.; Kobo, A.; Su, T.; Grunwald, A.; Green, O.; Nilsson, A. N.; Eisenberg,

E.; Ambjörnsson, T.; Westerlund, F.; Weinhold, E. ACS Nano 2016, 10, (11), 98239830.

33. Dai, M.; Jungmann, R.; Yin, P. Nat. Nanotechnol. 2016, 11, (9), 798-807.

34. Feroz, F.; Hobson, M.; Bridges, M. Mon. Not. of the R. Astron. Soc. 2009, 398, (4), 1601-1614.

35. Venta, K.; Shemer, G.; Puster, M.; Rodriguez-Manzo, J. A.; Balan, A.;

Rosenstein, J. K.; Shepard, K.; Drndic, M. ACS Nano 2013, 7, (5), 4629-4636.

36. Rodriguez-Manzo, J. A.; Puster, M.; Nicolai, A.; Meunier, V.; Drndic, M. ACS

Nano 2015, 9, (6), 6555-6564.

37. Carson, S.; Wilson, J.; Aksimentiev, A.; Wanunu, M. Biophys. J. 2014, 107, (10), 2381-93.

38. Garaj, S.; Hubbard, W.; Reina, A.; Kong, J.; Branton, D.; Golovchenko, J. Nature 2010, 467, (7312), 190.

39. $\quad$ Feng, J.; Liu, K.; Bulushev, R. D.; Khlybov, S.; Dumcenco, D.; Kis, A.; Radenovic, A. Nat. Nanotechnol. 2015, 10, (12), 1070.

40. Zhou, X.; Cai, S.; Hong, A.; You, Q.; Yu, P.; Sheng, N.; Srivannavit, O.;

Muranjan, S.; Rouillard, J. M.; Xia, Y. Nucleic Acids Res. 2004, 32, (18), 5409-5417. 


\section{Supporting Information}

\section{Digital Data Storage Using DNA Nanostructures and Solid-State Nanopores}

Kaikai Chen, Jinglin Kong, Jinbo Zhu, Niklas Ermann, Paul Predki, and Ulrich F. Keyser

\section{Table of contents}

Section 1. Synthesis and design of DNA carriers

Section 2. Nanopore characterization and measurement

Section 3. Data analysis for the DNA carriers with two hairpins

Section 4. Data analysis for the 56-bit and 112-bit DNA carriers 


\section{Section 1. Design and sequence of DNA carriers}

\subsection{Synthesis of DNA carriers}

We used linearized M13mp18 single-stranded DNA (ssDNA, 7228 bp after linearization, from

New England Biolabs) and complementary oligonucleotides (from Integrated DNA Technologies, abbreviated as oligos in the following) to synthesise DNA carriers. 190 complementary oligos fully occupy the 7228 bp distance with each length of 38 bp (except for the $46 \mathrm{bp}$ two ends, with 42 bp complementary sequences and 'TTTT' as the beginning and end). The oligos are numbered from 1 to 190 with sequences listed in Table S-1. Oligos with additional hairpins or ss DNA overhangs were used instead at designed positions to make protrusions on the carriers. Sequences start at 5' ends in this Supporting Information.

\begin{tabular}{|c|c|c|}
\hline UाIgo No. & sequence & Length (bp) \\
\hline$T$ & TITICGTAATCATGGICATAGCTGITTCCTGIGIGAAATTGITATC & 46 \\
\hline 2 & CGCTCACAATTCCACACAACATACGAGCCGGAAGCATA & 38 \\
\hline 3 & AAGTGIAAAGCCTGGGGTGCCIAATGAGIGAGCTAACT & 38 \\
\hline 4 & CACATIAATIGCGITGCGCICACTGCCCGCTITCCAGI & 38 \\
\hline 5 & CGGGAAACCIGTCGIGCCAGCIGCATIAATGAATCGGC & 38 \\
\hline 6 & CAACGCGCGGGGAGAGGCGGITIGCGIATIGGGCGCCA & 38 \\
\hline$T$ & GGGIGGITITICTITICACCAGIGAGACGGGCAACAGC & 38 \\
\hline 8 & TGATIGCCCITCACCGCCTGGCCCIGAGAGAGITGCAG & 38 \\
\hline 9 & CAAGCGGTCCACGCTGGTTIGCCCCAGCAGGCGAAAAT & 38 \\
\hline 10 & CCIGITIGATGGIGGITCCGAAATCGGCAAAATCCCTT & 38 \\
\hline$\pi$ & ATAAATCAAAAGAATAGCCCGAGATAGGGITGAGTGIT & 38 \\
\hline 12 & GIICCAGIIIGGAACAAGAGICCACTAITAAAGAACGT & 38 \\
\hline 13 & GGACTCCAACGTCAAAGGGCGAAAAACCGTCTATCAGG & 38 \\
\hline 14 & GCGATGGCCCACIACGTGAACCATCACCCAAATCAAGT & 38 \\
\hline 15 & TITITGGGGICGAGGTGCCGIAAAGCACIAAATCGGAA & 38 \\
\hline 16 & CCCTAAAGGGAGCCCCCGATITAGAGCITGACGGGGAA & 38 \\
\hline 17 & AGCCGGCGAACGTGGCGAGAAAGGAAGGGAAGAAAGCG & 38 \\
\hline 18 & AAAGGAGCGGGCGCIAGGGCGCIGGCAAGIGTAGCGGT & 38 \\
\hline 19 & CACGCIGCGCGIAACCACCACACCCGCCGCGCTIAATG & 38 \\
\hline 20 & CGCCGCIACAGGGCGCGIACIATGGITCLIIIGACGAG & 38 \\
\hline 21 & CACGTATAACGIGCTTTCCTCGTIAGAATCAGAGCGGG & 38 \\
\hline 22 & AGCTAAACAGGAGGCCGATIAAAGGGATTITAGACAGG & 38 \\
\hline 23 & AACGGIACGCCAGAATCCIGAGAAGIGITITIATAATC & 38 \\
\hline 24 & AGIGAGGCCACCGAGIAAAAGAGICIGICCAICACGCA & 38 \\
\hline 25 & AATTAACCGTTGIAGCAATACTTCTTIGATIAGTAATA & 38 \\
\hline 26 & ACATCACTIGCCTGAGTAGAAGAACTCAAACTATCGGC & 38 \\
\hline 21 & CTIGCIGGIAATATCCAGAACAATATIACCGCCAGCCA & 38 \\
\hline 28 & TIGCAACAGGAAAAACGCICAIGGAAATACC TACATIT & 38 \\
\hline 29 & TGACGCTCAATCGICIGAAATGGATIATITACATIGGC & 38 \\
\hline 30 & AGATICACCAGTCACACGACCAGTAATAAAAGGGACAT & 38 \\
\hline 31 & TCIGGCCAACAGAGATAGAACCCTICTGACCTGAAAGC & 38 \\
\hline 32 & GIAAGAAIACGIGGCACAGACAAIATIITIGAAIGGCT & 38 \\
\hline 33 & ATTAGTCTTTAATGCGCGAACTGATAGCCCTAAAACAT & 38 \\
\hline 34 & CGCCATIAAAAATACCGAACGAACCACCAGCAGAAGAT & 38 \\
\hline
\end{tabular}




\begin{tabular}{|c|c|c|}
\hline 35 & AAAACAGAGGTGAGGCGGTCAGTATTAACACCGCCTGC & 38 \\
\hline 36 & AACAGTGCCACGCTGAGAGCCAGCAGCAAATGAAAAAT & 38 \\
\hline 37 & CTAAAGCATCACCTTGCTGAACCTCAAATATCAAACCC & 38 \\
\hline 38 & $\begin{array}{l}\text { TCAATCAATATCTGGTCAGTTGGCAAATCAACAGTTGA } \\
\end{array}$ & 38 \\
\hline 39 & AAGGAATTGAGGAAGGTTATCTAAAATATCTTTAGGAG & 38 \\
\hline 40 & CACTAACAACTAATAGATTAGAGCCGTCAATAGATAAT & 38 \\
\hline 41 & ACATTTGAGGATTTAGAAGTATTAGACTTTACAAACAA & 38 \\
\hline 42 & $\begin{array}{l}\text { TTCGACAACTCGTATTAAATCCTTTGCCCGAACGTTAT } \\
\end{array}$ & 38 \\
\hline 43 & TAATTTTAAAAGTTTGAGTAACATTATCATTTTGCGGA & 38 \\
\hline 44 & ACAAAGAAACCACCAGAAGGAGCGGAATTATCATCATA & 38 \\
\hline 45 & $\begin{array}{l}\text { TTCCTGATTATCAGATGATGGCAATTCATCAATATAAT } \\
\end{array}$ & 38 \\
\hline 46 & CCTGATTGTTTGGATTATACTTCTGAATAATGGAAGGG & 38 \\
\hline 47 & TTAGAACCTACCATATCAAAATTATTTGCACGTAAAAC & 38 \\
\hline 48 & AGAAATAAAGAAATTGCGTAGATTTTCAGGTTTAACGT & 38 \\
\hline 49 & CAGATGAATATACAGTAACAGTACCTTTTACATCGGGA & 38 \\
\hline 50 & GAAACAATAACGGATTCGCCTGATTGCTTTGAATACCA & 38 \\
\hline 51 & AGTTACAAAATCGCGCAGAGGCGAATTATTCATTTCAA & 38 \\
\hline 52 & $\begin{array}{l}\text { TTACCTGAGCAAAAGAAGATGATGAAACAAACATCAAG } \\
\end{array}$ & 38 \\
\hline 53 & AAAACAAAATTAATTACATTTAACAATTTCATTTGAAT & 38 \\
\hline 54 & $\begin{array}{l}\text { TACCTTTTTTAATGGAAACAGTACATAAATCAATATAT } \\
\end{array}$ & 38 \\
\hline 55 & GTGAGTGAATAACCTTGCTTCTGIAAATCGTCGCTATT & 38 \\
\hline 56 & AATTAATTTTCCCTTAGAATCCTTGAAAACATAGCGAT & 38 \\
\hline 57 & AGCTTAGATTAAGACGCTGAGAAGAGTCAATAGTGAAT & 38 \\
\hline 59 & $\begin{array}{l}\text { TTATCAAAATCATAGGTCTGAGAGACTACCTTTTTAAC } \\
\end{array}$ & 38 \\
\hline 59 & CTCCGGCTTAGGTTGGGTTATATAACTATATGTAAATG & 38 \\
\hline 60 & CTGATGCAAATCCAATCGCAAGACAAAGAACGCGAGAA & 38 \\
\hline 61 & AACTTTTTCAAATATATTTTAGTTAATTTCATCTTCTG & 38 \\
\hline 62 & ACCTAAATTTAATGGTTTGAAATACCGACCGTGTGATA & 38 \\
\hline 63 & AATAAGGCGTTAAATAAGAATAAACACCGGAATCATAA & 38 \\
\hline 64 & $\begin{array}{l}\text { TTACTAGAAAAAGCCTGTTTAGTATCATATGCGTTATA } \\
\end{array}$ & 38 \\
\hline 65 & CAAATTCTTACCAGTATAAAGCCAACGCTCAACAGTAG & 38 \\
\hline 66 & GGCTTAATTGAGAATCGCCATATTTAACAACGCCAACA & 38 \\
\hline 67 & TGTAATTTAGGCAGAGGCATTTTCGAGCCAGTAATAAG & 38 \\
\hline 68 & AGAATATAAAGTACCGACAAAAGGTAAAGTAATTCTGT & 38 \\
\hline 69 & CCAGACGACGACAATAAACAACATGTTCAGCTAATGCA & 38 \\
\hline 70 & GAACGCGCCTGTTTATCAACAATAGATAAGTCCTGAAC & 38 \\
\hline 71 & AAGAAAAATAATATCCCATCCTAATTTACGAGCATGTA & 38 \\
\hline 72 & GAAACCAATCAATAATCGGCTGTCTTTCCTTATCATTC & 38 \\
\hline 73 & CAAGAACGGGTATTAAACCAAGTACCGCACTCATCGAG & 38 \\
\hline 74 & AACAAGCAAGCCGTTTTTATTTTCATCGTAGGAATCAT & 38 \\
\hline 75 & TACCGCGCCCAATAGCAAGCAAATCAGATATAGAAGGC & 38 \\
\hline 76 & $\begin{array}{l}\text { TTATCCGGTATTCTAAGAACGCGAGGCGTTTTAGCGAA } \\
\end{array}$ & 38 \\
\hline 77 & CCTCCCGACTTGCGGGAGGTTTTGAAGCCTTAAATCAA & 38 \\
\hline 78 & GATTAGTTGCTATTTTGCACCCAGCTACAATTTTATCC & 38 \\
\hline 79 & TGAATCTTACCAACGCTAACGAGCGTCTTTCCAGAGCC & 38 \\
\hline 80 & $\begin{array}{l}\text { TAATTTGCCAGTTACAAAATAAACAGCCATATTATTTA } \\
\end{array}$ & 38 \\
\hline 81 & $\begin{array}{l}\text { TCCCAATCCAAATAAGAAACGATTTTTTGTTTAACGTC } \\
\end{array}$ & 38 \\
\hline 82 & AAAAATGAAAATAGCAGCCTTTACAGAGAGAATAACAT & 38 \\
\hline 83 & AAAAACAGGGAAGCGCATTAGACGGGAGAATTAACTGA & 38 \\
\hline 84 & ACACCCTGAACAAAGTCAGAGGGTAATTGAGCGCTAAT & 38 \\
\hline 85 & ATCAGAGAGATAACCCACAAGAATTGAGTTAAGCCCAA & 38 \\
\hline 86 & TAATAAGAGCAAGAAACAATGAAATAGCAATAGCTATC & 38 \\
\hline 87 & TTACCGAAGCCCTTTTTAAGAAAAGTAAGCAGATAGCC & 38 \\
\hline 88 & GAACAAAGTTACCAGAAGGAAACCGAGGAAACGCAATA & 38 \\
\hline 89 & ATAACGGAATACCCAAAAGAACTGGCATGATTAAGACT & 38 \\
\hline 90 & CCTTATTACGCAGTATGTTAGCAAACGTAGAAAATACA & 38 \\
\hline 91 & TACATAAAGGTGGCAACATATAAAAGAAACGCAAAGAC & 38 \\
\hline 92 & ACCACGGAATAAGTTTATTTTGTCACAATCAATAGAAA & 38 \\
\hline 93 & ATTCATATGGTTTACCAGCGCCAAAGACAAAAGGGCGA & 38 \\
\hline 94 & CATTCAACCGATTGAGGGAGGGAAGGTAAATATTGACG & 38 \\
\hline 95 & GAAATTATTCATTAAAGGTGAATTATCACCGTCACCGA & 38 \\
\hline 96 & CTTGAGCCATTTGGGAATTAGAGCCAGCAAAATCACCA & 38 \\
\hline 97 & GTAGCACCATTACCATTAGCAAGGCCGGAAACGTCACC & 38 \\
\hline 98 & AATGAAACCATCGATAGCAGCACCGTAATCAGTAGCGA & 38 \\
\hline 99 & CAGAATCAAGITTGCCTTTAGCGICAGACTGTAGCGCG & 38 \\
\hline 100 & TTTTCATCGGCATTTTCGGTCATAGCCCCCTTATTAGC & 38 \\
\hline 101 & GTTTGCCATCTTTTCATAATCAAAATCACCGGAACCAG & 38 \\
\hline 102 & AGCCACCACCGGAACCGCCTCCCTCAGAGCCGCCACCC & 38 \\
\hline 103 & TCAGAACCGCCACCCTCAGAGCCACCACCCTCAGAGCC & 38 \\
\hline 104 & GCCACCAGAACCACCACCAGAGCCGCCGCCAGCATTGA & 38 \\
\hline 105 & CAGGAGGTTGAGGCAGGTCAGACGATTGGCCTTGATAT & 38 \\
\hline
\end{tabular}




\begin{tabular}{|c|c|c|}
\hline 106 & TCACAAACAAATAAATCCTCATTAAAGCCAGAATGGAA & 38 \\
\hline 107 & AGCGCAGTCTCTGAATTTACCGTTCCAGTAAGCGTCAT & 38 \\
\hline 108 & ACATGGCTTTTGATGATACAGGAGTGTACTGGTAATAA & 38 \\
\hline 109 & GTTTTAACGGGGTCAGTGCCTTGAGTAACAGTGCCCGT & 38 \\
\hline 110 & ATAAACAGTTAATGCCCCCTGCCTATTTCGGAACCTAT & 38 \\
\hline 111 & TATTCTGAAACATGAAAGTATTAAGAGGCTGAGACTCC & 38 \\
\hline 112 & TCAAGAGAAGGATTAGGATTAGCGGGGTTTTGCTCAGT & 38 \\
\hline 113 & ACCAGGCGGATAAGTGCCGTCGAGAGGGTTGATATAAG & 38 \\
\hline 114 & TATAGCCCGGAATAGG'GTATCACCGTACTCAGGAGGT & 38 \\
\hline 115 & TTAGTACCGCCACCCTCAGAACCGCCACCCTCAGAACC & 38 \\
\hline 116 & GCCACCCTCAGAGCCACCACCCTCATTTTCAGGGATAG & 38 \\
\hline 117 & CAAGCCCAATAGGAACCCATGTACCGTAACACTGAGTT & 38 \\
\hline 118 & TCGTCACCAGTACAAACTACAACGCCTGTAGCATTCCA & 38 \\
\hline 119 & CAGACAGCCCTCATAGTTAGCGTAACGATCTAAAGTTT & 38 \\
\hline 120 & TGTCGTCTTTCCAGACGTTAGTAAATGAATTTTCTGTA & 38 \\
\hline 121 & TGGGATTTTGCTAAACAACTTTCAACAGTTTCAGCGGA & 38 \\
\hline 122 & GTGAGAATAGAAAGGAACAACTAAAGGAATTGCGAATA & 38 \\
\hline 123 & ATAATTTTTTCACGTTGAAAATCTCCAAAAAAAAGGCT & 38 \\
\hline 124 & CCAAAAGGAGCCTTTAATTGTATCGGTTTATCAGCTTG & 38 \\
\hline 125 & CTTTCGAGGTGAATTTCTTAAACAGCTTGATACCGATA & 38 \\
\hline 126 & GTTGCGCCGACAATGACAACAACCATCGCCCACGCATA & 38 \\
\hline 127 & ACCGATATATTCGGTCGCTGAGGCTTGCAGGGAGTTAA & 38 \\
\hline 128 & AGGCCGCTTTTGCGGGATCGTCACCCTCAGCAGCGAAA & 38 \\
\hline 129 & GACAGCATCGGAACGAGGGTAGCAACGGCTACAGAGGC & 38 \\
\hline 130 & TTTGAGGACTAAAGACTT"T"TCATGAGGAAGTTTCCAT & 38 \\
\hline 131 & TAAACGGGTAAAATACGTAATGCCACTACGAAGGCACC & 38 \\
\hline 132 & AACCTAAAACGAAAGAGGCAAAAGAATACACTAAAACA & 38 \\
\hline 133 & CTCATCTTTGACCCCCAGCGATTATACCAAGCGCGAAA & 38 \\
\hline 134 & CAAAGTACAACGGAGATTTGTATCATCGCCTGATAAAT & 38 \\
\hline 135 & TGTGTCGAAATCCGCGACCTGCTCCATGTTACTTAGCC & 38 \\
\hline 136 & GGAACGAGGCGCAGACGGTCAATCATAAGGGAACCGAA & 38 \\
\hline 137 & CTGACCAACTTTGAAAGAGGACAGATGAACGGTGTACA & 38 \\
\hline 138 & GACCAGGCGCATAGGCTGGCTGACCTTCATCAAGAGTA & 38 \\
\hline 139 & ATCTTGACAAGAACCGGATATTCATTACCCAAATCAAC & 38 \\
\hline 140 & GTAACAAAGCTGCTCATTCAGTGAATAAGGCTTGCCCT & 38 \\
\hline 141 & GACGAGAAACACCAGAACGAGTAGTAAATTGGGCTTGA & 38 \\
\hline 142 & GATGGTTTAATTTCAACTT"TATCATTGTGAATTACCT & 38 \\
\hline 143 & TATGCGATTTTAAGAACTGGCTCATTATACCAGTCAGG & 38 \\
\hline 144 & ACGTTGGGAAGAAAAATCTACGTTAATAAAACGAACTA & 38 \\
\hline 145 & ACGGAACAACATTATTACAGGTAGAAAGATTCATCAGT & 38 \\
\hline 146 & TGAGATTTAGGAATACCACAT"TCAACTAATGCAGATAC & 38 \\
\hline 147 & ATAACGCCAAAAGGAATTACGAGGCATAGTAAGAGCAA & 38 \\
\hline 148 & CACTATCATAACCCTCGTTTACCAGACGACGATAAAAA & 38 \\
\hline 149 & CCAAAATAGCGAGAGGCTTTTGCAAAAGAAGTTTTGCC & 38 \\
\hline 150 & AGAGGGGG'AATAGTAAAATGITTAGACTGGATAGCGI & 38 \\
\hline 151 & CCAATACTGCGGAATCGTCATAAATATTCATTGAATCC & 38 \\
\hline 152 & CCCTCAAATGCTTTAAACAGTTCAGAAAACGAGAATGA & 38 \\
\hline 153 & CCATAAATCAAAAATCAGGTCTTTACCCTGACTATTAT & 38 \\
\hline 154 & AGTCAGAAGCAAAGCGGATTGCATCAAAAAGATTAAGA & 38 \\
\hline 155 & GGAAGCCCGAAAGACTTCAAATATCGCGTTTTAATTCG & 38 \\
\hline 156 & AGCTTCAAAGCGAACCAGACCGGAAGCAAACTCCAACA & 38 \\
\hline 157 & GGTCAGGATTAGAGAGTACCTTTAATTGCTCCTTTTGA & 38 \\
\hline 158 & TAAGAGGTCATTTT TGCGGATGGCTTAGAGCTTAATTG & 38 \\
\hline 159 & CTGAATATAATGCTGTAGCTCAACATGTTTTAAATATG & 38 \\
\hline 160 & CAACTAAAGTACGGTGTCTGGAAGTTTCATTCCATATA & 38 \\
\hline 161 & ACAGTTGATTCCCAATTCTGCGAACGAGTAGATTTAGT & 38 \\
\hline 162 & TTGACCATTAGATACATTTCGCAAATGGTCAATAACCT & 38 \\
\hline 163 & GTTTAGCTATATTTTCATTTGGGGCGCGAGCTGAAAAG & 38 \\
\hline 164 & GTGGCATCAATTCTACTAATAGTAGTAGCATTAACATC & 38 \\
\hline 165 & CAATAAATCATACAGGCAAGGCAAAGAATTAGCAAAAT & 38 \\
\hline 166 & TAAGCAATAAAGCCTCAGAGCATAAAGCTAAATCGGTT & 38 \\
\hline 167 & GTACCAAAAACATTATGACCCTGTAATACTTTTGCGGG & 38 \\
\hline 168 & AGAAGCCTTTATTTCAACGCAAGGATAAAAATTTTTAG & 38 \\
\hline 169 & AACCCTCATATATTTTAAATGCAATGCCTGAGTAATGT & 38 \\
\hline 170 & GTAGGTAAAGATTCAAAAGGGIGAGAAAGGCCGGAGAC & 38 \\
\hline 171 & AGTCAAATCACCATCAATATGATATTCAACCGTTCTAG & 38 \\
\hline 172 & CTGATAAATTAATGCCGGAGAGGGTAGCTATTTTTGAG & 38 \\
\hline 173 & AGATCTACAAAGGCTATCAGGTCATTGCCTGAGAGTCT & 38 \\
\hline 174 & GGAGCAAACAAGAGAATCGATGAACGG'AAATCGIAAAA & 38 \\
\hline 175 & CTAGCATGTCAATCATATGTACCCCGGTTGATAATCAG & 38 \\
\hline 176 & AAAAGCCCCAAAAACAGGAAGATTGTATAAGCAAATAT & 38 \\
\hline
\end{tabular}




\begin{tabular}{|c|c|c|}
\hline 177 & TTAAATTGTAAACGTTAATATTTTGTTAAAATTCGCAT & 38 \\
\hline 178 & TAAATTTTTGTTAAATCAGCTCATTTTTTAACCAATAG & 38 \\
\hline 179 & GAACGCCATCAAAAATAATTCGCGTCTGGCCTTCCTGT & 38 \\
\hline 180 & AGCCAGCTTTCATCAACATTAAATGTGAGCGAGTAACA & 38 \\
\hline 181 & ACCCGICGGATTCTCCGIGGGAACAAACGGCGGATTGA & 38 \\
\hline 182 & CCGTAATGGGATAGGTCACGTTGGTGTAGATGGGCGCA & 38 \\
\hline 183 & TCGTAACCGTGCATCTGCCAGTTTGAGGGGACGACGAC & 38 \\
\hline 184 & AGTATCGGCCTCAGGAAGATCGCACTCCAGCCAGCTTT & 38 \\
\hline 185 & CCGGCACCGCTTCTGGTGCCGGAAACCAGGCAAAGCGC & 38 \\
\hline 186 & CATTCGCCATTCAGGCTGCGCAACTGTTGGGAAGGGCG & 38 \\
\hline 187 & ATCGGTGCGGGCCTCTTCGCTATTACGCCAGCTGGCGA & 38 \\
\hline 188 & AAGGGGGATGTGCTGCAAGGCGATTAAGTTGGGTAACG & 38 \\
\hline 189 & CCAGGGTTTTCCCAGTCACGACGTTGTAAAACGACGGC & 38 \\
\hline 190 & CAGTGCCAAGCTTGCATGCCTGCAGGTCGACTCTAGAGGATCTTTT & 46 \\
\hline
\end{tabular}

Table S-1. Sequences of the 190 complementary oligos.

The DNA carrier synthesis is introduced in a former study. ${ }^{1}$ The carrier was made by mixing together $8 \mu \mathrm{L}$ M13mp18 DNA (100 nM), $20 \mu \mathrm{L}$ oligonucleotide mix (each oligo $200 \mathrm{nM}), 4 \mu \mathrm{L}$ $100 \mathrm{mM} \mathrm{MgCl} 2,1.2 \mu \mathrm{L} 100 \mathrm{mM}$ Tris- $\mathrm{HCl}(\mathrm{pH}=8), 10 \mathrm{mM}$ EDTA, and $6.8 \mu \mathrm{L}$ deionised water. Then the mixture was heated to $70^{\circ} \mathrm{C}$ followed by a linear cooling ramp to $25^{\circ} \mathrm{C}$ over $50 \mathrm{~min}$. After annealing, these excess oligonucleotides were removed using Amicon Ultra $100 \mathrm{kDa}$ filters. One tube annealed as above was added with $460 \mu \mathrm{L}$ of $10 \mathrm{mM}$ Tris- $\mathrm{HCl}(\mathrm{pH}=8), 0.5 \mathrm{mM} \mathrm{MgCl} 2$ and centrifuged at $9000 \mathrm{~g}$ for $10 \mathrm{~min}$ at $4^{\circ} \mathrm{C} .460 \mu \mathrm{L}$ more $10 \mathrm{mM}$ Tris- $\mathrm{HCl}(\mathrm{pH}=8), 0.5 \mathrm{mM} \mathrm{MgCl} 2$ was added and the sample was centrifuged again for $10 \mathrm{~min}$. The sample was then recovered by turning the filter upside down and centrifuging for $2 \mathrm{~min}$ at $1000 \mathrm{~g}$. The solution was immediately added after filtering to make the final concentration $10 \mathrm{mM}$ Tris- $\mathrm{HCl}, 100 \mathrm{mM} \mathrm{NaCl}, 2 \mathrm{mM} \mathrm{MgCl}$.

Designs of protrusions on DNA carriers are shown in the following parts.

\subsection{Design of the DNA carrier with one 16 bp and one 8 bp hairpins}

This was made by replacing the Oligo 94 and Oligo 142 with Oligo $16 \mathrm{H} 94$ and Oligo 8H142 shown below with red and blue parts marked to form the hairpins.

Oligo $16 \mathrm{H} 94$ 
CATTCAACCGATTGAGGGAGGGAAGGTAAATATTGACG

TTTTCGCCTGCACTCTACCTTTTGGTAGAGTGCAGGCGA

Oligo $8 \mathrm{H} 142$

GATGGTTTAATTTCAACTTTAATCATTGTGAATTACCT TTTAGAGGAGGTTTTCCTCCTCT

\subsection{Design of the DNA carrier with two 16 bp hairpins with a distance of 76 bp}

The Oligo 94 and Oligo 96 were replaced with Oligo 16H94 and Oligo 16H96 shown below.

Oligo $16 \mathrm{H} 94$

CATTCAACCGATTGAGGGAGGGAAGGTAAATATTGACG

TTTTCGCCTGCACTCTACCTTTTGGTAGAGTGCAGGCGA

Oligo $16 \mathrm{H} 96$

CTTGAGCCATTTGGGAATTAGAGCCAGCAAAATCACCA TTTCGCCTGCACTCTACCTTTTGGTAGAGTGCAGGCGA

\subsection{Design of the 56-bit and 112-bit DNA carriers}

In this design, the Oligos $13,16,19, \ldots 178$ (increase by 3,56 in total) were replaced with strands consisting of 16 or $8 \mathrm{bp}$ hairpins and $38 \mathrm{bp}$ parts to bind to the scaffold. The sequences are given in Tables S-2 and S-3.

\begin{tabular}{|c|c|c|}
\hline Name & Sequence & $\begin{array}{l}\text { Replaced } \\
\text { oligo No. }\end{array}$ \\
\hline $16 \mathrm{H} 37$ & CTAAAGCATCACCTTGCTGAACCTCAAATATCAAACCC TTTTCGCCTGCACTCTACCTTTTGGTAGAGTGCAGGCGA & 37 \\
\hline $16 \mathrm{H} 40$ & $\begin{array}{l}\text { CACTAACAACTAATAGATTAGAGCCGTCAATAGATAAT TTTTCGCCTGCACTCTACCTTTTGGTAGAGTGCAGGCGA } \\
\end{array}$ & 40 \\
\hline $16 \mathrm{H} 43$ & TAATTTTAAAAGTTTGAGTAACATTATCATTTTGCGGA TTTTCGCCTGCACTCTACCTTTTGGTAGAGTGCAGGCGA & 43 \\
\hline $16 \mathrm{H} 46$ & CCTGATTGTTGGATTATACTTCTGAATAATGGAAGGG TTTTCGCCTGCACTCTACC TTT TGGTAGAGTGCAGGCGA & 46 \\
\hline $16 \mathrm{H} 49$ & CAGATGAATATACAGTAACAGTACCTTTTACATCGGGA TTTTCGCCTGCACTCTACCTTTTGGTAGAGTGCAGGCGA & 49 \\
\hline $16 \mathrm{H} 52$ & TTACCTGAGCAAAAGAAGATGATGAAACAAACATCAAG TTTTCGCCTGCACTCTACCTTTTGGTAGAGTGCAGGCGA & 52 \\
\hline $16 \mathrm{H} 55$ & $\begin{array}{l}\text { GTGAGTGAATAACCTTGCTTCTGTAAATCGTCGCTATT TTTTCGCCTGCACTCTACCTTTTGGTAGAGTGCAGGCGA } \\
\end{array}$ & 55 \\
\hline $16 \mathrm{H} 58$ & TTATCAAAATCATAGGTCTGAGAGACTACCTTTTTAAC TTTTCGCCTGCACTCTACCTTTTGGTAGAGTGCAGGCGA & 58 \\
\hline $16 \mathrm{H} 64$ & $\begin{array}{l}\text { TTACTAGAAAAAGCCTGTTTAGTATCATATGCGTTATA TTTTCGCCTGCACTCTACCTTTTGGTAGAGTGCAGGCGA } \\
\end{array}$ & 64 \\
\hline $16 \mathrm{H} 76$ & TTATCCGGTATTCTAAGAACGCGAGGCGTTTTAGCGAA TTTTCGCCTGCACTCTACCTTTTGGTAGAGTGCAGGCGA & 76 \\
\hline $16 \mathrm{H} 85$ & ATCAGAGAGATAACCCACAAGAATTGAGTTAAGCCCAA TTTTCGCCTGCACTCTACCTTTTGGTAGAGTGCAGGCGA & 85 \\
\hline
\end{tabular}




\begin{tabular}{|c|c|c|}
\hline $16 \mathrm{H} 88$ & CGCCTGCACTCTACCTTTTGGTAGAGTGCAGGCGA & 88 \\
\hline $16 \mathrm{H} 91$ & $\begin{array}{l}\text { TACATAAAGGTGGCAACATATAAAAGAAACGCAAAGAC TTTTCGCCTGCACTCTACCTTTTGGTAGAGTGCAGGCGA } \\
\end{array}$ & 91 \\
\hline 16H94 & CATTCAACCGATTGAGGGAGGGAAGGTAAATATTGACG TTTTCGCCTGCACTCTACCTTTTGGTAGAGTGCAGGCGA & 94 \\
\hline 16H97 & GTAGCACCATTACCATTAGCAAGGCCGGAAACGTCACC TTTTCGCCTGCACTCTACCTTTTGGTAGAGTGCAGGCGA & 97 \\
\hline $16 \mathrm{H} 100$ & TTTTCATCGGCATTTTCGGTCATAGCCCCCTTATTAGC TTTTCGCCTGCACTCTACCTTTTGGTAGAGTGCAGGCGA & 100 \\
\hline $16 \mathrm{H} 103$ & TCAGAACCGCCACCCTCAGAGCCACCACCCTCAGAGCC TTTTCGCCTGCACTCTACCTTTTGGTAGAGTGCAGGCGA & 103 \\
\hline $16 \mathrm{H} 106$ & $\begin{array}{l}\text { TCACAAACAAATAAATCCTCATTAAAGCCAGAATGGAA TTTTCGCCTGCACTCTACCTTTTGGTAGAGTGCAGGCGA } \\
\end{array}$ & 106 \\
\hline $16 \mathrm{H} 112$ & TCAAGAGAAGGATTAGGATTAGCGGGGTTTTGCTCAGT TTTTCGCCTGCACTCTACCTTTTGGTAGAGTGCAGGCGA & 112 \\
\hline $16 \mathrm{H} 115$ & TTAGTACCGCCACCCTCAGAACCGCCACCCTCAGAACC TTTTCGCCTGCACTCTACCTTTTGGTAGAGTGCAGGCGA & 115 \\
\hline $16 \mathrm{H} 121$ & TGGGATTTTGCTAAACAACTTTCAACAGTTTCAGCGGA TTTTCGCCTGCACTCTACCTTTTGGTAGAGTGCAGGCGA & 121 \\
\hline $16 \mathrm{H} 124$ & CCAAAAGGAGCCTTTAATTGTATCGGTTTATCAGCTTG TTTTCGCCTGCACTCTACCTTTTGGTAGAGTGCAGGCGA & 124 \\
\hline $16 \mathrm{H} 127$ & ACCGATATATTCGGTCGCTGAGGCTTGCAGGGAGTTAA TTTTCGCCTGCACTCTACCTTTTGGTAGAGTGCAGGCGA & 127 \\
\hline $16 \mathrm{H} 130$ & TTTGAGGACTAAAGACTTTTTCATGAGGAAGTTTCCAT TTTTCGCCTGCACTCTACCTTTTGGTAGAGTGCAGGCGA & 130 \\
\hline $16 \mathrm{H} 157$ & GGTCAGGATTAGAGAGTACCTTTAATTGCTCCTTTTGA TTTTCGCCTGCACTCTACCTTTTGGTAGAGTGCAGGCGA & 157 \\
\hline $16 \mathrm{H} 160$ & CAACTAAAGTACGGTGTCTGGAAGTTTCATTCCATATA TTTTCGCCTGCACTCTACCTTTTGGTAGAGTGCAGGCGA & 160 \\
\hline $16 \mathrm{H} 163$ & $\begin{array}{l}\text { GTTTAGCTATATTTTCATTTGGGGCGCGAGCTGAAAAG TTTTCGCCTGCACTCTACCTTTTGGTAGAGTGCAGGCGA } \\
\end{array}$ & 163 \\
\hline $16 \mathrm{H} 166$ & TAAGCAATAAAGCCTCAGAGCATAAAGCTAAATCGGTT TTTTCGCCTGCACTCTACCTTTTGGTAGAGTGCAGGCGA & 166 \\
\hline $16 \mathrm{H} 169$ & AACCCTCATATATTTTAAATGCAATGCCTGAGTAATGT TTTTCGCCTGCACTCTACCTTTTGGTAGAGTGCAGGCGA & 169 \\
\hline $16 \mathrm{H} 172$ & CTGATAAATTAATGCCGGAGAGGGTAGCTATTTTTGAG TTTTCGCCTGCACTCTACCTTTTGGTAGAGTGCAGGCGA & 172 \\
\hline $16 \mathrm{H} 175$ & CTAGCATGTCAATCATATGTACCCCGGTTGATAATCAG TTTTCGCCTGCACTCTACCTTTTGGTAGAGTGCAGGCGA & 175 \\
\hline $16 \mathrm{H} 178$ & TAAATTTTTGTTAAATCAGCTCATTTTTTAACCAATAG TTTTCGCCTGCACTCTACCTTTTGGTAGAGTGCAGGCGA & 178 \\
\hline
\end{tabular}

Table S-2. Oligos with 16 bp hairpins for the 56-bit DNA carrier.

\begin{tabular}{|c|c|c|}
\hline Name & Sequence & Replaced oligo No. \\
\hline $8 \mathrm{H} 13$ & GGACTCCAACGTCAAAGGGCGAAAAACCGTCTATCAGG TTTAGAGGAGGTTTTCCTCCTCT & 13 \\
\hline $8 \mathrm{H} 16$ & CCCTAAAGGGAGCCCCCGATTTAGAGCTTGACGGGGAA TTTAGAGGAGGTTTTCCTCCTCT & 16 \\
\hline $8 \mathrm{H} 19$ & CACGCTGCGCGTAACCACCACACCCGCCGCGCTTAATG TTTAGAGGAGGTTTTCCTCCTCT & 19 \\
\hline $8 \mathrm{H} 22$ & AGCTAAACAGGAGGCCGATTAAAGGGATTTTAGACAGG TTTAGAGGAGGTTTTCCTCCTCT & 22 \\
\hline $8 \mathrm{H} 25$ & AATTAACCGTTGTAGCAATACTTCTTTGATTAGTAATA TTTAGAGGAGGTTTTCCTCCTCT & 25 \\
\hline $8 \mathrm{H} 28$ & TTGCAACAGGAAAAACGCTCATGGAAATACCTACATTT TTTAGAGGAGGTTTTCCTCCTCT & 28 \\
\hline $8 \mathrm{H} 31$ & TCTGGCCAACAGAGATAGAACCCTTCTGACCTGAAAGC TTTAGAGGAGGTTTTCCTCCTCT & 31 \\
\hline $8 \mathrm{H} 34$ & CGCCATTAAAAATACCGAACGAACCACCAGCAGAAGAT TTTAGAGGAGGTTTTCCTCCTCT & 34 \\
\hline $8 \mathrm{H} 6 \mathrm{l}$ & AACTTTTTCAAATATATTTTAGTTAATTTCATCTTCTG TTTAGAGGAGGTTTTCCTCCTCT & 61 \\
\hline $8 \mathrm{H} 67$ & TGTAATTTAGGCAGAGGCATTTTCGAGCCAGTAATAAG TTTAGAGGAGGTTTTCCTCCTCT & 67 \\
\hline $8 \mathrm{H70}$ & GAACGCGCCTGTTTATCAACAATAGATAAGTCCTGAAC TTTAGAGGAGGTTTTCCTCCTCT & 70 \\
\hline $8 \mathrm{H73}$ & CAAGAACGGGTATTAAACCAAGTACCGCACTCATCGAG TTTAGAGGAGGTTTTCCTCCTCT & 73 \\
\hline $8 \mathrm{H} 79$ & TGAATCTTACCAACGCTAACGAGCGTCTTTCCAGAGCC TTTAGAGGAGGTTTTCCTCCTCT & 79 \\
\hline $8 \mathrm{H} 82$ & AAAAATGAAAATAGCAGCCTTTACAGAGAGAATAACAT TTTAGAGGAGGTTTTCCTCCTCT & 82 \\
\hline $8 \mathrm{H} 109$ & GTTTTAACGGGGTCAGTGCCTTGAGTAACAGTGCCCGT TTTAGAGGAGGTTTTCCTCCTCT & 109 \\
\hline $8 \mathrm{H} 118$ & TCGTCACCAGTACAAACTACAACGCCTGTAGCATTCCA TTTAGAGGAGGTTTTCCTCCTCT & 118 \\
\hline $8 \mathrm{H} 133$ & CTCATCTTTGACCCCCAGCGATTATACCAAGCGCGAAA TTTAGAGGAGGTTTTCCTCCTCT & 133 \\
\hline $8 \mathrm{H136}$ & GGAACGAGGCGCAGACGGTCAATCATAAGGGAACCGAA TTTAGAGGAGGTTTTCCTCCTCT & 136 \\
\hline $8 \mathrm{H139}$ & ATCTIGACAAGAACCGGATATTCATTACCCAAATCAAC TTTAGAGGAGGT"TTCCTCCICT & 139 \\
\hline $8 \mathrm{H} 142$ & GATGGTTTAATTTCAACTTTAATCATTGTGAATTACCT TTTAGAGGAGGTTTTCCTCCTCT & 142 \\
\hline $8 \mathrm{H} 145$ & ACGGAACAACATTATTACAGGTAGAAAGATTCATCAGT TTTAGAGGAGGTTTTCCTCCTCT & 145 \\
\hline $8 \mathrm{H} 148$ & CACTATCATAACCCTCGTTTACCAGACGACGATAAAAA TTTAGAGGAGGTTTTCCTCCTCT & 148 \\
\hline $8 \mathrm{H} 151$ & CCAATACTGCGGAATCGTCATAAATATTCATTGAATCC TTTAGAGGAGGTTTTCCTCCTCT & 151 \\
\hline $8 \mathrm{H} 154$ & AGTCAGAAGCAAAGCGGATTGCATCAAAAAGATTAAGA TTTAGAGGAGGTTTTCCTCCTCT & 154 \\
\hline
\end{tabular}

Table S-3. Oligos with 8 bp hairpins for the 56-bit DNA carrier.

The 112-bit DNA carrier was formed by linking two types of 56-bit DNA carriers. For the two types, everything is the same as shown above except that the first oligos were replaced with the linkers (Linkers A and B) shown below. Then the link was achieved by mixing two samples and keeping at room temperature for $1 \mathrm{~h}$. 
Linker A

AAGTCCTCGTCGGTCGCG CGTAATCATGGTCATAGCTGTTTCCTGTGTGAAATTGTTATC

Linker B

CGCGACCGACGAGGACTT CGTAATCATGGTCATAGCTGTTTCCTGTGTGAAATTGTTATC 


\section{Section 2. Nanopore characterization and measurement}

The fabrication parameters, scanning electron microscopy (SEM) images, model and calculations and example data are shown below.

\subsection{Nanopore fabrication parameters}

We used one-line programs for the fabrication of the nanopores. The parameters are shown below. Please refer to the P-2000 manual ${ }^{2}$ for the explanation of the parameters.

HEAT $=575$, FIL $=0$, VEL $=25$, DEL $=170$, PUL $=225$

\subsection{SEM images}

An example nanopore scanning electron microscope (SEM) image is shown in Figure S-1. The inner diameter was estimated by the outer diameter of the quartz capillary considering that the ratio of the inner diameter to outer diameter kept constant during fabrication.

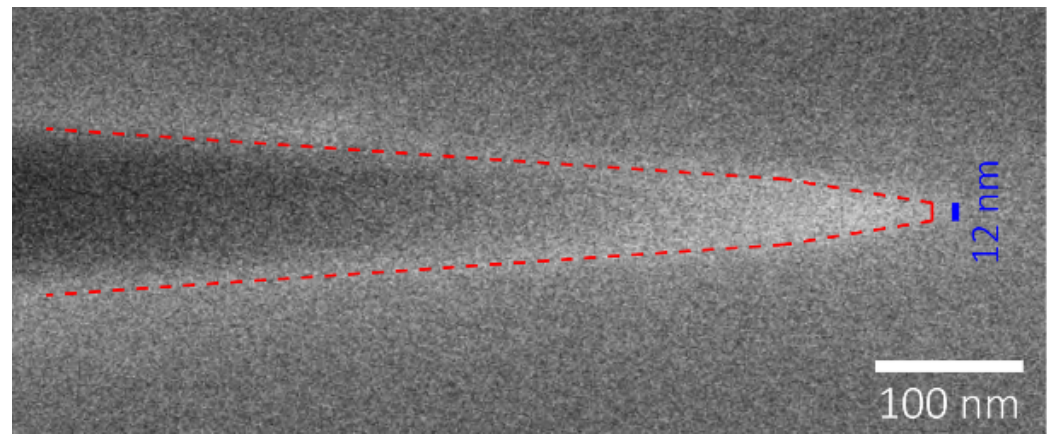

Figure S-1. An example SEM image of the nanopore. Scale bar $=100 \mathrm{~nm}$. The blue bars show the outer diameters. 


\subsection{Model and calculations}

We model the geometry of the pores as two parts with two different conical angles - the part close to the tip with length $L$ and the other with infinite length (Figure S-2).

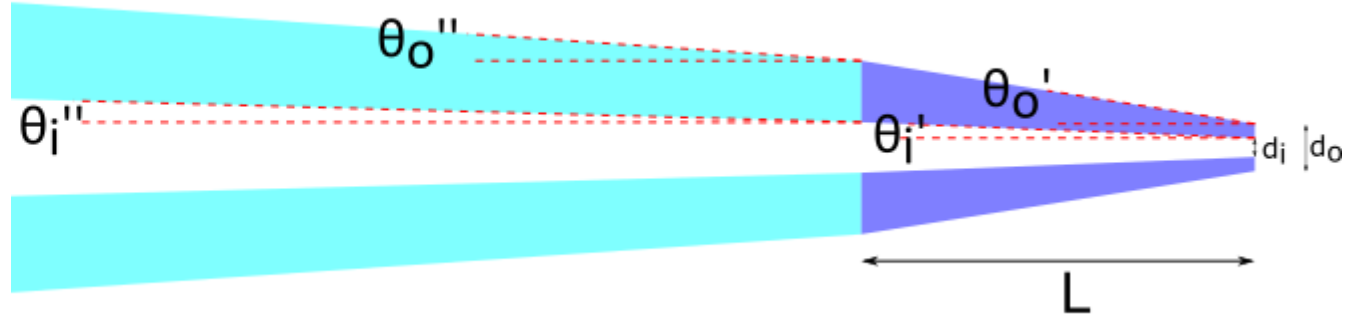

Figure S-2. Model of the geometry of the glass nanopores. The two parts with different conical angles are marked in different colours.

The inner conical angles are $\theta_{\mathrm{i}}^{\prime}$ and $\theta_{\mathrm{i}}{ }^{\prime \prime}$ and outer conical angles are $\theta_{0}{ }^{\prime}$ and $\theta_{0}{ }^{\prime \prime}$ respectively. $d_{\mathrm{i}}$ and $d_{\mathrm{o}}$ are the inner and outer diameters.

We then calculate the ionic current through the pore with and without DNA blocking it.

The resistance of the pore without DNA is calculated as ${ }^{3}$

$$
R_{\text {Nanopore }}=\frac{4 L}{\sigma \pi d_{i}\left(d_{i}+2 \operatorname{Ltan} \theta_{i}^{\prime}\right)}+\frac{2}{\sigma \pi\left(d_{i}+2 \operatorname{Ltan} \theta_{i}^{\prime}\right) \tan \theta_{i}^{\prime \prime}}+\frac{1}{2 \sigma d_{i}}
$$

where $\sigma$ is the conductivity of the solution.

The inner diameter and conical angles are inferred from the outer values considering the ratio of the inner diameter to outer diameter is constant, and are calculated as

$$
\begin{aligned}
d_{i} & =0.4 d_{o} \\
\tan \theta_{i}^{\prime} & =0.4 \tan \theta_{o}^{\prime}
\end{aligned}
$$




$$
\tan \theta_{i}^{\prime \prime}=0.4 \tan \theta_{o}^{\prime \prime}
$$

where $0.4(0.2 / 0.5)$ is the ratio.

When the DNA is in the pore, the resistance is then ( $a$ is the DNA radius)

$$
\begin{aligned}
R_{\text {Nanopore with DNA }=} & \frac{1}{2 \sigma \pi \tan \theta_{i}^{\prime}}\left(\ln \frac{2 \operatorname{Ltan} \theta_{i}^{\prime}+d_{i}-2 a}{2 \operatorname{Ltan} \theta_{i}^{\prime}+d_{i}+2 a}-\ln \frac{d_{i}-2 a}{d_{i}+2 a}\right) \\
& -\frac{1}{2 \sigma \pi a \tan \theta_{i}^{\prime \prime}} \ln \frac{2 \operatorname{Ltan} \theta_{i}^{\prime}+d_{i}-2 a}{2 \operatorname{Ltan} \theta_{i}^{\prime}+d_{i}+2 a}+\frac{1}{2 \sigma d_{i}}
\end{aligned}
$$

Substituting in the values we estimate from the SEM images, the current values can be calculated. The conductivity of $4 \mathrm{M} \mathrm{LiCl}$ of $\sigma=18.4 \mathrm{~S} / \mathrm{m}$ and the DNA radius of $a=1.25 \mathrm{~nm}$ are used.

For the $\sim 5 \mathrm{~nm}$ diameter nanopores

$d_{\mathrm{i}}=5 \mathrm{~nm}, L=100 \mathrm{~nm}, \theta_{\mathrm{i}}^{\prime}=0.064 \mathrm{rad}, \theta_{\mathrm{i}}{ }^{\prime}=0.027 \mathrm{rad}$

The current values at $400 \mathrm{mV}$ without DNA and with DNA are calculated as $2.51 \mathrm{nA}$ and $2.32 \mathrm{nA}$, with the difference of $0.18 \mathrm{nA}$. The calculated current values agree with our experimental data shown below.

\subsection{Nanopore measurement}

The nanopores were integrated into Polydimethylsiloxane (PDMS) chips (Figure S-3). 

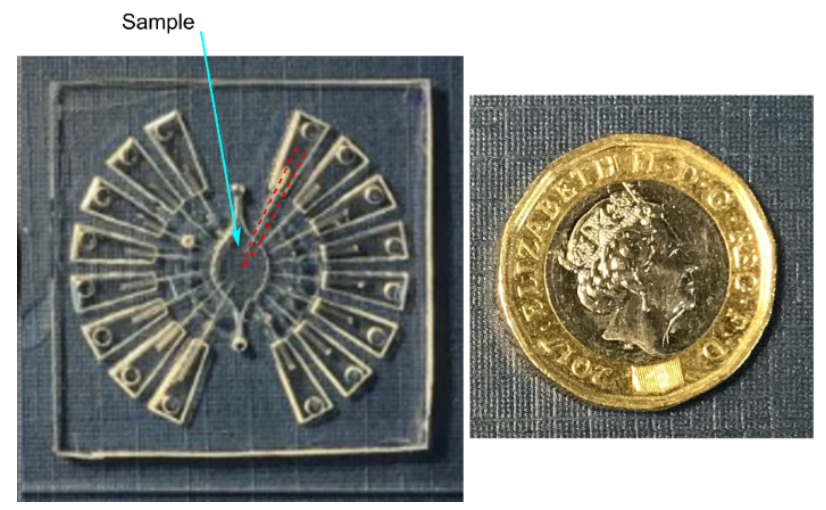

Figure S-3. A nanopore chip integrated with 16 nanopores. The coin (one British Pound) shows the scale. An example channel is marked by red dashed lines.

IV curves of the pores are measured in $4 \mathrm{M} \mathrm{LiCl}$ solution and shown in Figure $\mathrm{S}-4$.

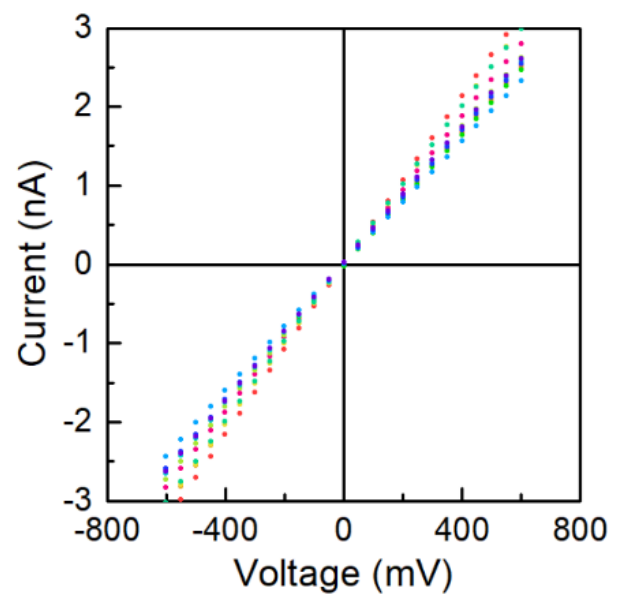

Figure S-4. IV curves of the $\sim 5 \mathrm{~nm}$ diameter nanopores in $4 \mathrm{M} \mathrm{LiCl}$ solution. 12 pores were measured.

Examples of a current trace and typical events are shown in Figure S-5. 


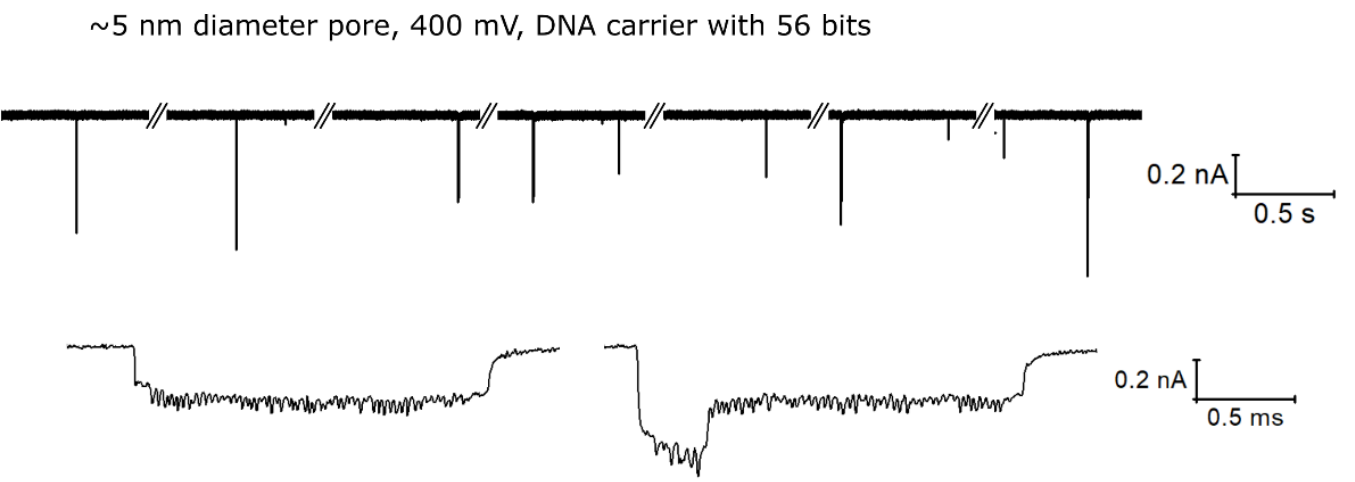

Figure S-5. An example current trace and example events. An example current trace and example events caused by the 56-bit carriers at $400 \mathrm{mV}$ measured with a $\sim 5 \mathrm{~nm}$ diameter nanopore. 


\section{Section 3. Data analysis for the DNA carrier with two hairpins}

\subsection{Data analysis for the DNA carrier with two hairpins, one 16 bp and one 8 bp}

The data was analysed with a peak finding algorithm ${ }^{4}$. Single unfolded events were picked to extract the amplitudes of the secondary current drop caused by the 16 and 8 bp hairpins. Example events are shown in Figure S-6.

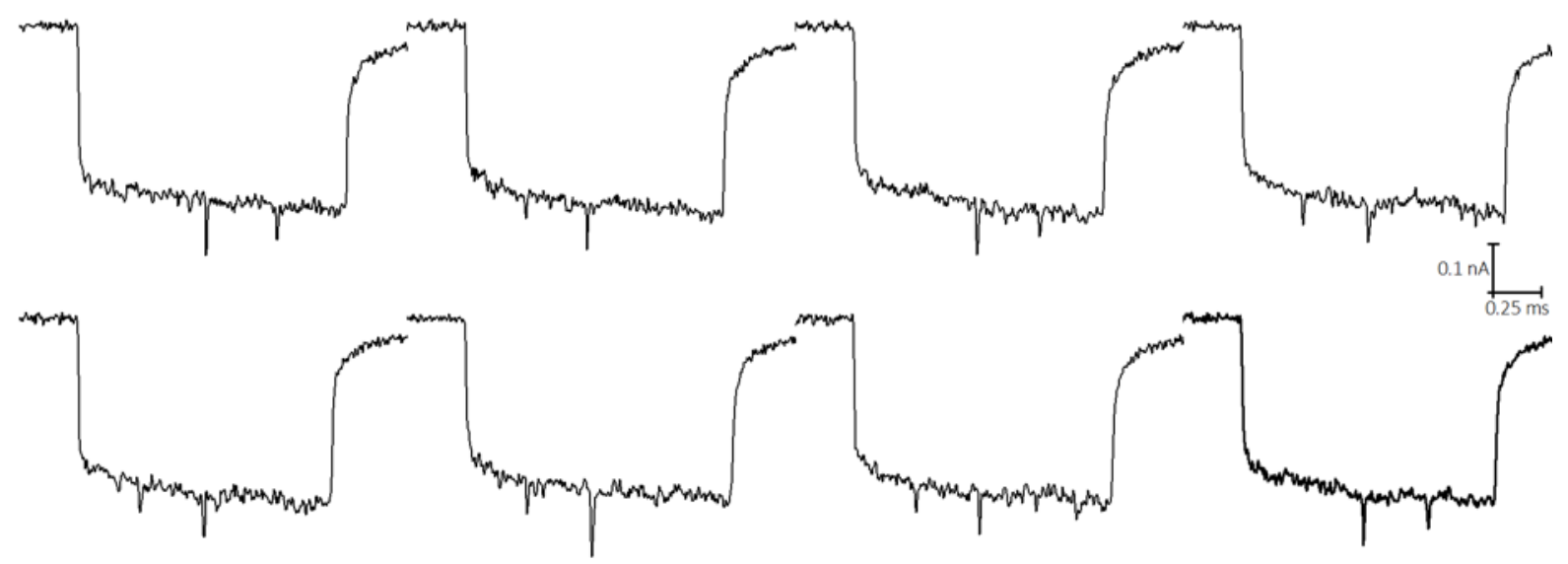

Figure S-6. Example events caused by the DNA carrier with one 16 bp and one 8 bp hairpins.

\subsection{Data analysis for the DNA carrier with two close 16 hairpins}

Three nanopores were used for the measurement with example events shown in Figure S-7 and statistics shown in Table S-4. 


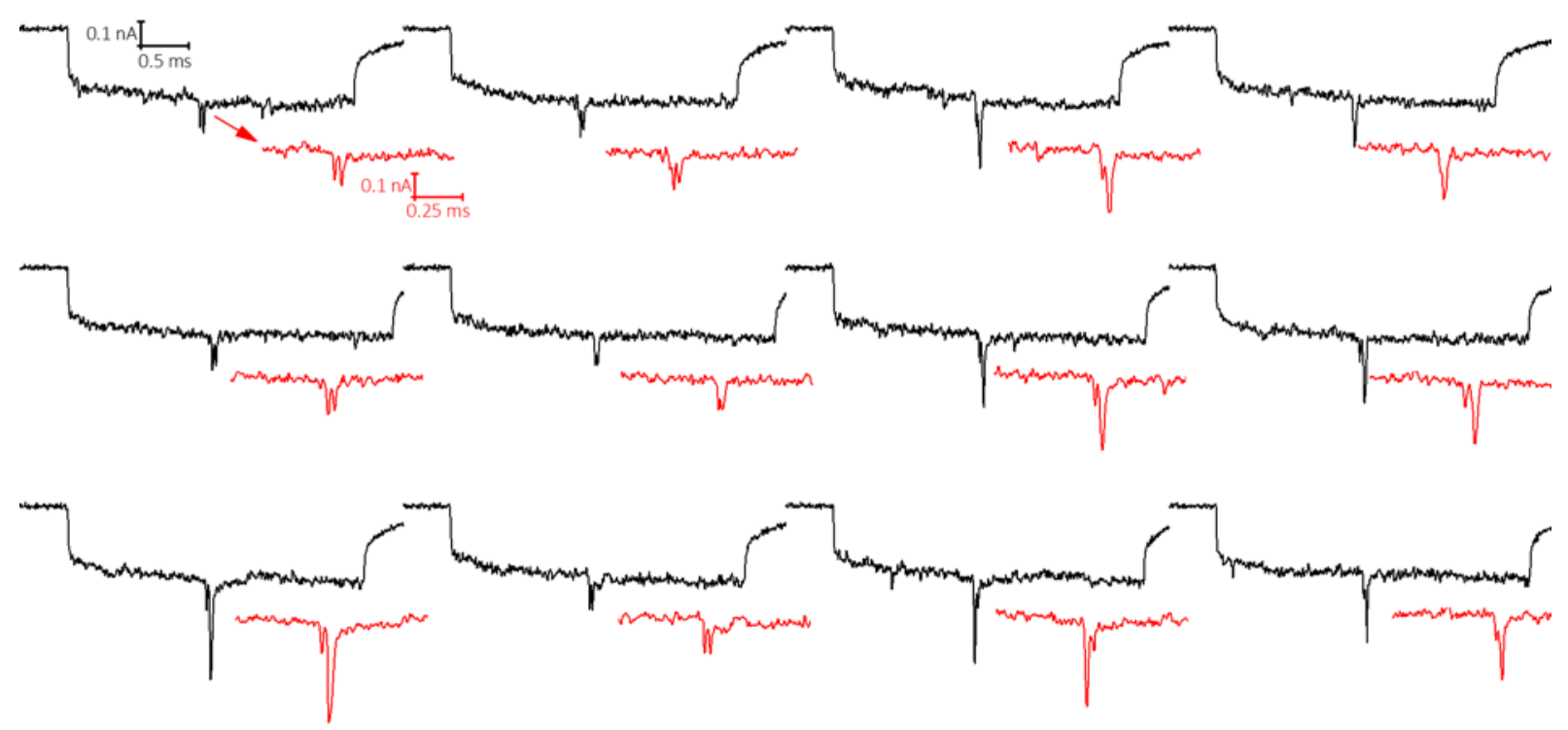

Figure S-7. Example events caused by the DNA carrier with two 16 bp hairpins with a 114 bp distance. Red plots show the zoom in details.

\begin{tabular}{|c|c|c|c|c|c|}
\hline No. & Pore name & Unfolded event No. & Readable event No. & Resolved event No. & Percentage of the resolved event (\%) \\
\hline 1 & $76 \_$A & 90 & 88 & 68 & 77.3 \\
\hline 2 & $76 \_B$ & 116 & 109 & 50 & 45.9 \\
\hline 3 & $76 \_C$ & 108 & 103 & 51 & 49.5 \\
\hline
\end{tabular}

Table S-4. Statistics of the events from 3 pores measured with the DNA carrier with two 16 bp hairpins. 


\section{Section 4. Data analysis for the 56-bit and 112-bit DNA carriers}

\subsection{Example events and statistics}

We measured the 56-bit and 112-bit DNA carriers with two nanopores. The sample was made by mixing 56-bit DNA carriers with the linking ends. Not all the carriers were linked together so we had both 56-bit and 112-bit carriers in the solution. In the nanopore measurement, we had 103 unfolded events in total, with 66 of monomers (56-bit carriers) and 37 dimers (112-bit carriers) separated by the translocation durations. A preselected algorithm was used to assess the events with 58 monomers and 19 dimers retained for the bit analysis. Example events are shown in Figures S-8 and S-9.

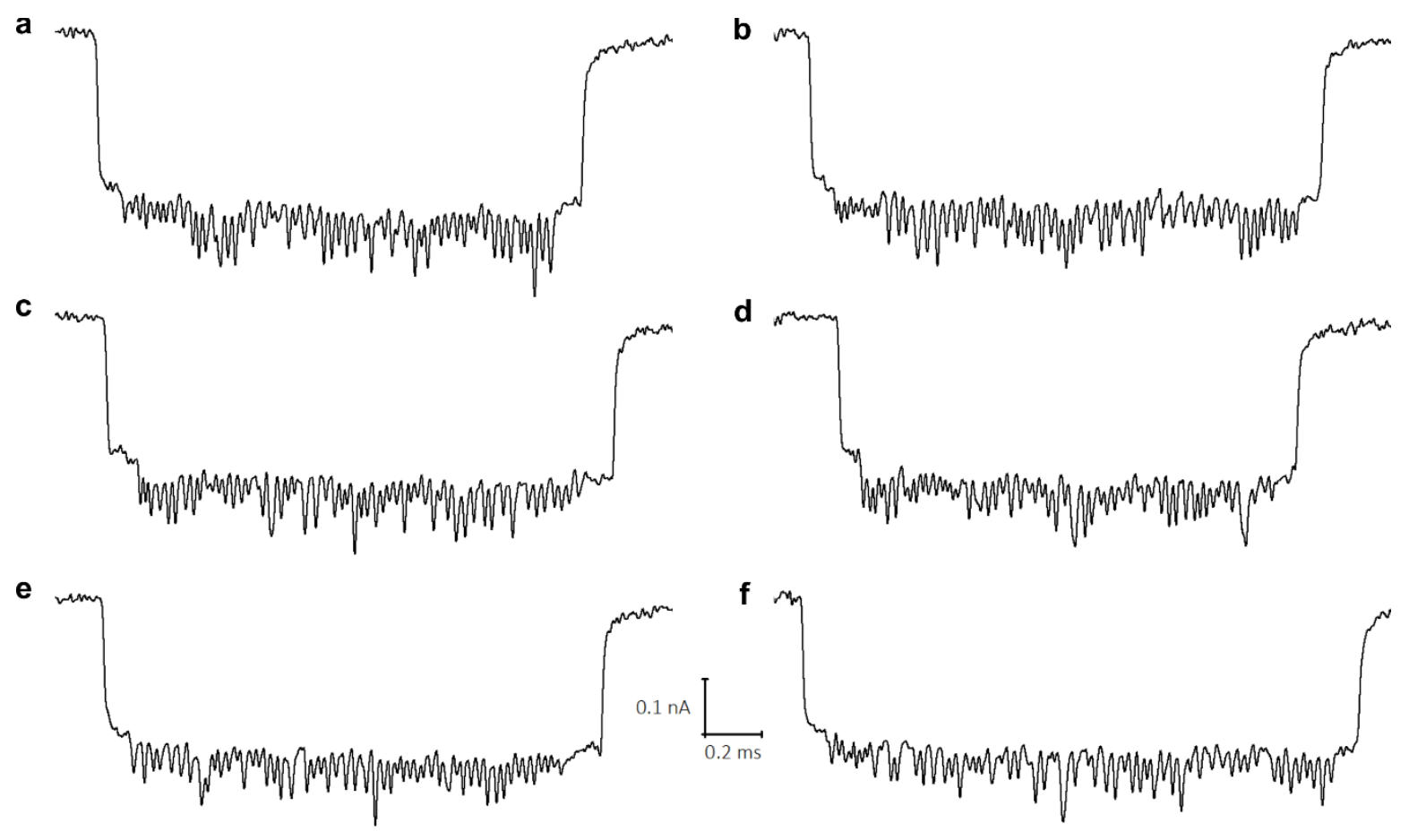

Figure S-8. Examples of events caused by the 56-bit DNA carriers. 
a

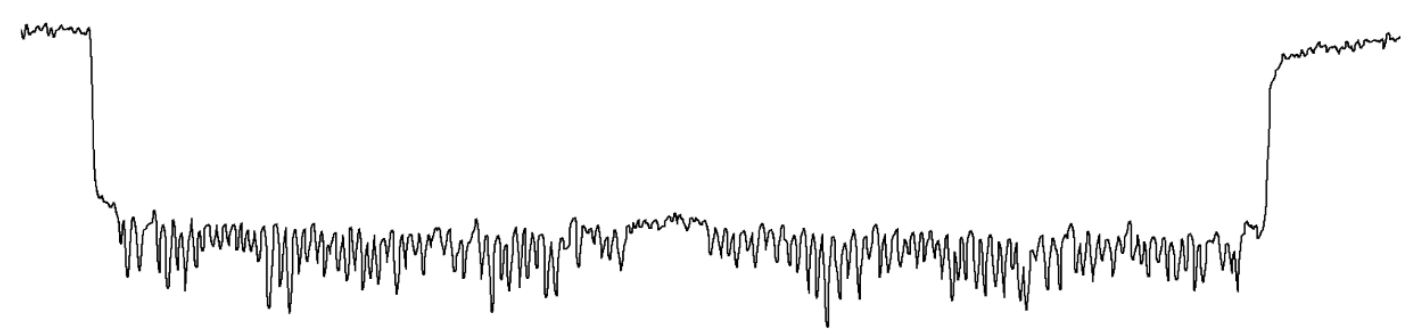

b

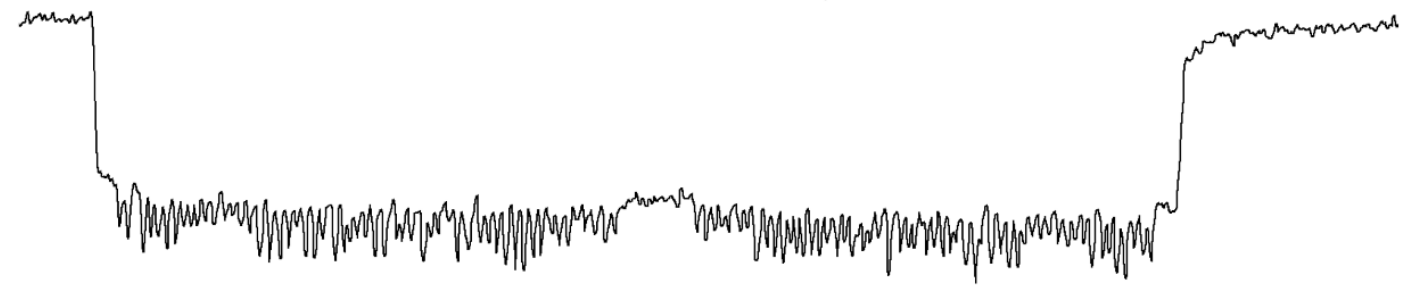

C

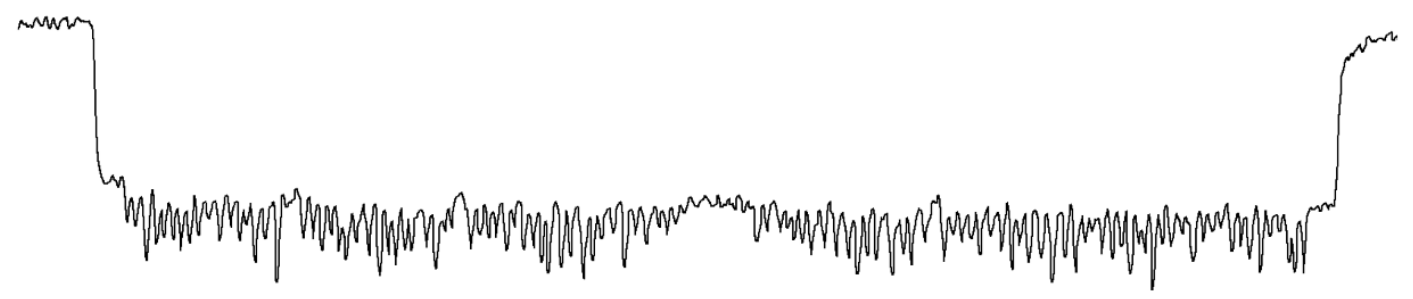

d

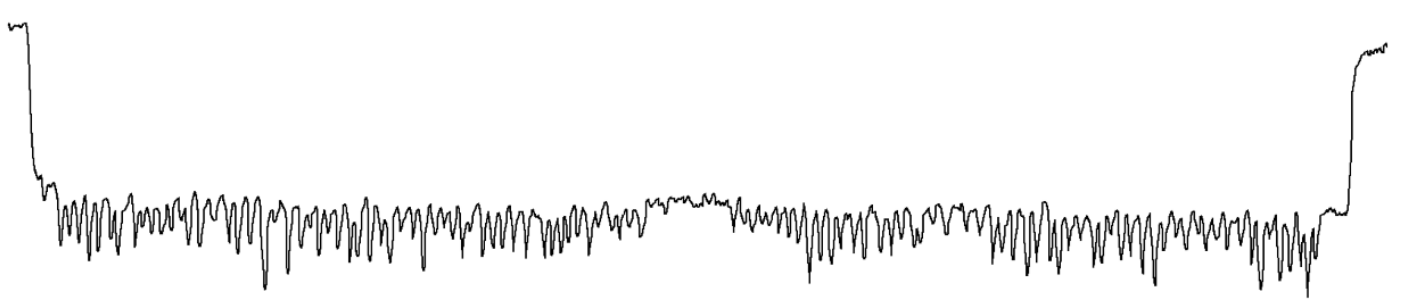

e

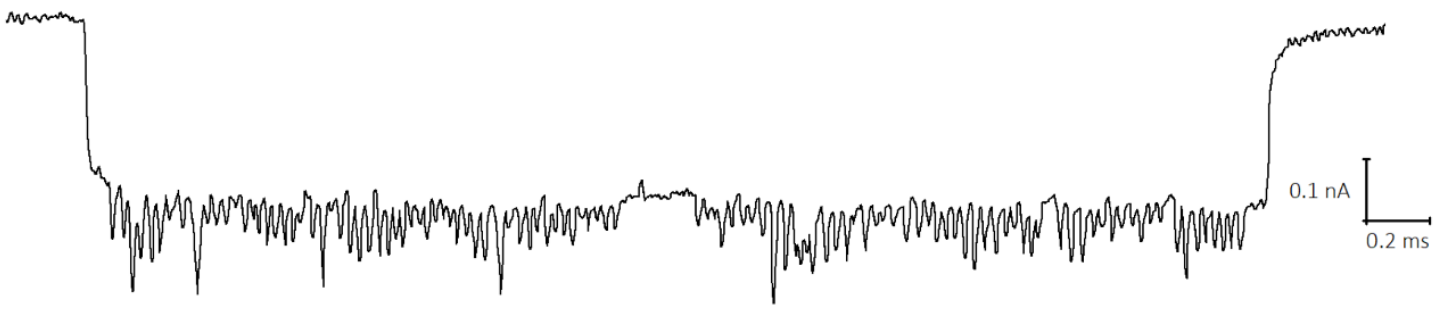

Figure S-9. Examples of events caused by the 112-bit DNA carriers.

\subsection{Data analysis with the Bayesian inference method}

Bayesian techniques emerge from a fundamental statement about conditional probabilities called Bayes' rule. ${ }^{5}$ The Bayesian inference method for nanopore data analysis will be discussed in an upcoming paper from our group. 


\section{References}

1. Bell, N.A.W. \& Keyser, U.F. Digitally encoded DNA nanostructures for multiplexed, single-molecule protein sensing with nanopores. Nat. Nanotechnol. 11, 645-651 (2016).

2. P-2000 laser-based micropipette puller system operation manual REV. 2.4 (20120105).

3. Bell, N.A. \& Keyser, U.F. Specific protein detection using designed DNA carriers and nanopores. J. Am. Chem. Soc. 137, 2035-2041 (2015).

4. Chen, K. et al. Ionic Current-Based Mapping of Short Sequence Motifs in Single DNA Molecules Using Solid-State Nanopores. Nano Lett. 17, 5199-5205 (2017).

5. Gelman, A., Carlin, J.B., Stern, H.S. \& Rubin, D.B. Bayesian Data Analysis Second edition Corrected version (30 Jan 2008). (1995). 